\title{
Dark sector impact on gravitational collapse of an electrically charged scalar field
}

\author{
Anna Nakonieczna, ${ }^{a, b}$ Marek Rogatko ${ }^{a}$ and tukasz Nakonieczny ${ }^{c}$ \\ ${ }^{a}$ Institute of Physics, Maria Curie-Sktodowska University, \\ Plac Marii Curie-Sktodowskiej 1, 20-031 Lublin, Poland \\ ${ }^{b}$ Institute of Agrophysics, Polish Academy of Sciences, \\ Doświadczalna 4, 20-290 Lublin, Poland \\ ${ }^{c}$ Institute of Theoretical Physics, Faculty of Physics, University of Warsaw, \\ Pasteura 5, 02-093 Warszawa, Poland \\ E-mail: aborkow@kft.umcs.lublin.pl, rogat@kft.umcs.lublin.pl, \\ Lukasz.Nakonieczny@fuw.edu.pl
}

ABSTRACT: Dark matter and dark energy are dominating components of the Universe. Their presence affects the course and results of processes, which are driven by the gravitational interaction. The objective of the paper was to examine the influence of the dark sector on the gravitational collapse of an electrically charged scalar field. A phantom scalar field was used as a model of dark energy in the system. Dark matter was modeled by a complex scalar field with a quartic potential, charged under a U(1)-gauge field. The dark components were coupled to the electrically charged scalar field via the exponential coupling and the gauge field-Maxwell field kinetic mixing, respectively. Complete non-linear simulations of the investigated process were performed. They were conducted from regular initial data to the end state, which was the matter dispersal or a singularity formation in a spacetime. During the collapse in the presence of dark energy dynamical wormholes and naked singularities were formed in emerging spacetimes. The wormhole throats were stabilized by the violation of the null energy condition, which occurred due to a significant increase of a value of the phantom scalar field function in its vicinity. The square of mass parameter of the dark matter scalar field potential controlled the formation of a Cauchy horizon or wormhole throats in the spacetime. The joint impact of dark energy and dark matter on the examined process indicated that the former decides what type of an object forms, while the latter controls the amount of time needed for the object to form. Additionally, the dark sector suppresses the natural tendency of an electrically charged scalar field to form a dynamical Reissner-Nordström spacetime during the gravitational collapse.

KEYwords: Classical Theories of Gravity, Black Holes

ARXIV EPRINT: 1508.02657 


\section{Contents}

1 Introduction 1

2 Theoretical model of the evolution $\quad 6$

3 Details of computer simulations and results analysis $\quad 12$

4 Electrically charged scalar field collapse with dark energy $\quad \mathbf{1 5}$

$\begin{array}{lll}\text { 4.1 Dynamical emergence of wormhole-like structures and naked singularities } & 15\end{array}$

$\begin{array}{lll}4.2 & \text { Vicinity of wormhole throats } & 18\end{array}$

$\begin{array}{lll}4.3 & \text { Black hole-wormhole duality } & 20\end{array}$

4.4 Properties of wormholes 20

5 Influence of dark matter on electrically charged scalar field evolution $\quad 22$

$\begin{array}{lll}5.1 & \text { Spacetime structures } & 23\end{array}$

5.2 Fields behavior in spacetimes 24

$\begin{array}{ll}\text { 5.3 Characteristics of the formed black holes } & 27\end{array}$

6 Collective effect of dark components on the considered collapse $\quad 27$

$\begin{array}{lll}6.1 & \text { Spacetime structures } & 27\end{array}$

$\begin{array}{lll}6.2 & \text { Fields behavior in spacetimes } & 29\end{array}$

$\begin{array}{lll}6.3 & \text { Properties of the emerging objects } & 31\end{array}$

7 Conclusions $\quad 33$

$\begin{array}{ll}\text { A Numerical computations } & 36\end{array}$

\section{Introduction}

Black holes which exist in the Universe are rotating and electrically neutral, due to the presence of an interstellar medium. They are formed during gravitational collapse of a star, whose mass exceeds the Tolman-Oppenheimer-Volkoff limit [1, 2]. One of theoretical toy models of this process, which allows to describe the resulting spacetime structures and properties of the emerging objects, is a dynamical evolution of an electrically charged scalar field $[3,4]$. A considerable advantage of its investigations is that they allow to describe physics of both outer and inner regions of the arising objects.

Dynamical gravitational collapse attracts scientific interest also because the structures of dynamically formed spacetimes differ significantly from their non-dynamical counterparts. This means that the knowledge of static solutions, which describe a particular system, is insufficient to characterize the results of dynamical processes, which take place 
within it. For this reason, the evolution of a massless electrically charged scalar field under the influence of gravity was examined $[5,6]$. It turned out that the dynamical ReissnerNordström spacetime is substantially different from the static one due to the existence of a central spacelike singularity and a null singularity along the Cauchy horizon, which results from the mass inflation phenomenon [7]. The effect of pair creation during the collapse and the subsequent evaporation of a black hole were also considered [3, 4, 8, 9]. The collapse of a Brans-Dicke field was elaborated in [10-13], while the results of the gravitational evolution in Einstein-Maxwell-dilaton theory with standard and phantom couplings of the involved fields were described in [14-16].

According to current cosmic microwave background (CMB) measurements performed by Planck, the amount of dark matter and dark energy in the Universe is about $27 \%$ and $69 \%$, respectively [17]. Such high abundance of the dark components, both of which interact gravitationally, undoubtedly affects the course and results of the gravitational collapse, which was one of the motivations for the analyses presented in the paper. The conducted research concerning the dark sector influence on the relatively small-scale process supplement vast investigations on its significance during the cosmological evolution.

Until now, the collapse in the presence of the dark sector was studied mainly in the context of the large scale structure formation and the course of a few astrophysical processes. The evolution of dark matter concentration in the form of a spherically symmetric dust cloud of a finite radius was examined on a background spacetime containing dark energy modeled by isotropic and anisotropic fluids, as well as the Chaplygin gas [18-20]. The structure formation during the evolution of quintessence dark energy induced by the collapse of dark matter halos was described in [21]. The spherical collapse model of the structure formation on cosmological scales in the presence of the dark sector was also considered [22, 23]. The evolution was followed on a background of a flat, homogeneous and isotropic universe, while the matter content was modeled by a multicomponent fluid. The clustering process was investigated during the non-linear spherical 'top hat' collapse of the generalized Chaplygin gas, which unifies dark energy and dark matter into a single matter component [24].

Recently, the role of the dark sector in gravitational collapse has been studied from the astrophysical perspective. The issue of a neutron star collapse due to capture and sedimentation of dark matter within its core was considered as a solution to two interesting observational puzzles [25]. One of them are fast radio bursts, which are probably extragalactic, transient bright radio pulses of an unexplained origin [26]. The second is a missing pulsar problem, which is related to the non-detection of pulsars within the inner core of the Galactic Center of the radius close to 10 parsecs [27]. The early stage and the longterm phase of the formation of supermassive black holes progenitors via a direct gas collapse in dark matter halos at high redshifts was studied in [28, 29].

The above-mentioned analyses were mostly focused on the behavior of matter during its gravitational collapse. The research was performed within the framework of fluid dynamics on either fixed or evolving background spacetimes. The current studies, by contrast, involve examining dynamical matter-geometry systems with the matter content of spacetime described in terms of classical field theory. To our knowledge, such a methodology has not yet been used in the analyses of the role of the dark sector in gravitational collapse. 
It allowed us not only to investigate the matter behavior, but also to follow the geometry dynamics observed during the evolution. The latter was reflected in the obtained spacetime structures. Due to the fact that they resulted from the fully non-linear computations conducted within the whole spacetime region subjected to the collapse dynamics, they were not limited by the capabilities of the previously employed local techniques $[18,19]$ and allowed presenting global characteristics of the forming spacetimes. Their interpretation enabled us to describe causal relations between distinct spacetime areas and to test the geometrical properties of both outer and inner regions of the emerging objects.

The first indirect evidence suggesting the existence of an electromagnetically non-interacting unknown matter component of the Universe came from observations of the galactic rotation curves [30, 31]. Since then, the concept of dark matter has put down its roots in modern cosmology. Apart from the aforementioned experiments, the gravitational interaction of dark matter with the remaining content of the Universe may be inferred from the observations of $\mathrm{CMB}$, baryon acoustic oscillations (BAO), the rate of the large scale structures formation [17, 32] or galactic collisions, like the Bullet Cluster [33]. These observations also provide information of the dark matter abundance and distribution in the Universe, as well as its non-gravitational nature, i.e., possible interaction types and the lifetime of the dark matter particle. Unfortunately, they are insufficient to fully identify the particle nature of dark matter, which is a dominant matter component of the Universe. However, the non-gravitational interactions of dark matter disfavoring some of the proposed extensions of the Standard Model were revealed recently [34, 35].

There is yet another branch of modern physics that is immensely interested in the topic, namely particle physics. The Standard Model of particle physics, despite being very successful in describing the visible matter, lacks any dark matter candidates. From this perspective the quest of gathering and analyzing information about dark matter is the quest of understanding the beyond-Standard Model physics. This situation and an enormous technological advancement triggered in the last few decades made the subject of dark matter one of the most interesting in modern physics. Astrophysical observations (other than these mentioned above) provide hints that dark matter may be responsible for the $\gamma$-ray bursts from galactic centers [36-38] and keV photons from galactic clusters [39, 40]. The terrestrial experiments in supercolliders such as the Large Hadron Collider (LHC) also strive to identify dark matter or at least constrain its models [41], for a current list of the dark matter models proposed to search for in the LHC run-2 data see [42]. Apart from the above two sources of the information on dark matter, a new one appeared recently. It is concerned with the theoretical investigation of the role of dark matter in the missing pulsar problem $[25,43]$ and its impact on the composition and lifetime of the early generation of stars [44].

As a dark matter model we used one of the viable, from the particle physics perspective, models of the dark matter sector. It is composed of a complex scalar field with a quartic self-interaction, which is charged under an additional Abelian gauge field. Its coupling to the standard matter sector (represented by an electrically charged scalar field) was implemented via a kinetic mixing between the introduced gauge field and the electromagnetic field [45]. A new experiment constraining such a coupling is currently under preparation [46], while some previous results can be found, e.g., in [47]. The considered 
model describes one or two dark matter candidates, depending on the vacuum expectation value (vev) of the complex scalar field. If the vev is non-zero, the dark matter candidate is the massive gauge boson mentioned earlier (in the particle physics literature often called $Z^{\prime}$ or dark photon). When the scalar does not possess the vev the candidate can be either the scalar or the gauge boson. A slightly modified version of this model was analyzed in the context of dark matter phenomenology $[48,49]$ and the GeV-scale $\gamma$-ray excess in the Galactic Center [50]. On the other hand, a new theoretical tool for detecting the influence of the dark matter sector on characteristic quantities of holographic superconductors was proposed [51-53]. It happens that the coupling constant of the dark matter sector to the ordinary Maxwell field imprints its existence during holographic phase transitions and these phenomena may constitute a possible way of understanding the nature of the dark matter sector.

Dark energy is a notion assigned within the $\Lambda \mathrm{CDM}$ cosmological model to the content of the Universe, which remains after determining the amount of matter detectable either directly (photons, neutrinos and baryonic matter) or indirectly (dark matter). It is responsible for the present-day accelerated expansion of the Universe [54-57]. Experimental restrictions on dark energy models are imposed by observations of supernovae Ia, combined with the CMB and BAO measurements [58]. The obtained constraints allow of the existence of dark energy in the form of phantom matter [59]. Its pressure $P$ and density $\rho$ satisfy the relation $P<-\rho$, which means that the value of its barotropic index $w=P \rho^{-1}$ is less than -1 . Matter of this type is coupled repulsively to gravity and thus exerts negative pressure and causes cosmic acceleration. Such a phantom scalar field coupled exponentially to the electrically charged scalar field was chosen as a dark energy model in our research.

Investigating a dynamical evolution in the presence of phantom fields is also justified due to a wide variety of non-dynamical solutions to Einstein equations involving such fields. New classes of spherically symmetric solutions of Einstein-Maxwell-dilaton theory with a phantom coupling were considered in $[60,61]$, while static multicentered solutions in this theory were discussed in [62]. A vast array of diversified and complex solutions opens new possibilities of finding their interesting dynamical counterparts.

The concept of a static wormhole was introduced by Wheeler [63] and extended by Morris and Thorne [64], who invented a class of potentially traversable wormholes. The wormhole throat is kept open by the presence of exotic matter, whose energy-momentum tensor violates the energy conditions. A scalar field coupled to gravity in a phantom manner is a good candidate for such a type of matter, which supports wormhole traversability [65]. The quoted works initiated studies of wormholes as topological bridges connecting two distinct regions of spacetime. There were proposed various models such as thin shells in cosmological background [66], in Brans-Dicke gravity [67], in the low-energy limit of the string theory [68], as well as wormholes supported by scalar phantom fields [69, 70], tachyonic matter [71] or non-linear electrodynamics [72]. Recently, to circumvent the problem of exotic matter, the modified-gravity theories were considered as suitable candidates to obtain stable traversable wormholes [73-76]. It is worth emphasizing that these researches deal with constructing eternal, i.e., non-dynamical wormholes. There also exists a research branch, which is concerned with physics of evolving wormholes [77-79]. The aim of our studies 
was to test the dynamical formation of such objects during fully non-linear investigations of the gravitational collapse.

Because of the ambiguous nature of wormholes in the view of astrophysical observations, a considerable resurgence of interests in their physics was observed recently. Experimental studies of phenomena and processes which result from the presence of a black hole in spacetime are based on the effects related to its external gravitational field. Wormholes possess the same gravitational properties as black holes, which are characteristic for them from the observational viewpoint [80]. For this reason, a wormhole is an alternative to a black hole and hence various possibilities of the experimental distinction between these two types of objects are being analyzed [81, 82].

Apart from the efforts to observationally distinguish wormholes from black holes, the issue of experimental distinction between naked singularities and black holes has also been pursued. These two types of objects could be differentiated through their gravitational lensing features [83], such as a number of generated images of a particular light source [84], their orientation in space, total magnifications and time delays [85, 86]. Another possibility is investigating the properties of accretion disks, which form around naked singularities and black holes, as their luminosities and angular velocities of the particles within them depend on the type of the central object [87, 88]. The above findings indicated that the observational consequences of the existence of naked singularities and black holes differ. It opens a possibility of testing the cosmic censorship hypothesis experimentally through astronomical measurements [89].

Astrophysical objects present in the Universe are dynamical, as they constantly undergo changes due to interactions with the surrounding environment. Thus one of the elements necessary to achieve the aforementioned goal, that is observational determination of a true nature of the astrophysical objects with strong gravitational fields, is to investigate the formation and behavior of black holes, wormholes and naked singularities during dynamical evolutions.

The leading research objective of the current studies was to test the influence of dark energy and dark matter on the collapse of an electrically charged scalar field. The role of the dark sector during the analyzed process was investigated through the prism of the forming spacetime structures, the behavior of fields within them and properties of the emerging dynamical objects. The paper is organized as follows. In section 2 we present the theoretical model, which enables us to study the dynamical gravitational collapse of an electrically charged scalar field accompanied by the dark sector. Section 3 contains basic information on solving the derived equations of motion and particulars of the results presentation. In sections 4 and 5 we discuss the course and outcomes of the investigated process running in the presence of dark energy and dark matter, respectively. Section 6 is devoted to studies of the joint influence of these two components on the evolution. The summary of the obtained results and prospects of further research are placed in section 7. Comments on numerical methods used for solving the equations of motion and the code accuracy checks can be found in appendix A. 


\section{Theoretical model of the evolution}

The model used to investigate the dynamical collapse of interest consists of three parts, whose construction is based on self-interacting scalar fields. The first one refers to a complex scalar field $\psi$ coupled with the Maxwell field $A_{\mu}$. The second one is a phantom scalar field $\phi$, which represents dark energy in the system. Its exponential coupling to the previous field is consistent with the low-energy string theory regime. The third component is a massive complex scalar field $\chi$ with a quartic potential coupled with a U(1)-gauge field $P_{\mu}$, which is coupled to the Maxwell field. It serves as dark matter in the system. Since the model incorporates notions taken from string theory, it is convenient to write the most general form of the action in the string frame

$$
\hat{S}=\int d^{4} x \sqrt{-\hat{g}}\left\{e^{-2 \phi}\left[\hat{R}-2 \xi(\hat{\nabla} \phi)^{2}+e^{2 \alpha \phi} \hat{\mathcal{L}}_{S F}\right]+\hat{\mathcal{L}}_{D M}\right\},
$$

where the constant $\xi$ determines the nature of the coupling between $\phi$ and gravity. It is set as equal to -1 , which means that the field is phantom [61,62]. The constant $\alpha$ characterizes the coupling between the scalar field $\phi$ and the electrically charged one. Since the lowenergy limit of the string theory is considered, we set $\alpha$ as equal to -1 [90]. This value was incorporated in the equations below. The dark energy and dark matter sectors are chosen to be independent, so dark matter represented by the Lagrangian $\hat{\mathcal{L}}_{D M}$ is not coupled to the phantom scalar field via the exponential coupling $e^{-2 \phi}$. The presented model enables us to study the impact of dark components on the evolution both separately and jointly. Throughout the computations, we used the geometrized units system, in which $8 \pi G=c=1$.

The Lagrangian of the electrically charged scalar field $\psi$ is given by the expression

$$
\hat{\mathcal{L}}_{S F}=-\frac{1}{2} \hat{D}_{\beta} \psi\left(\hat{D}^{\beta} \psi\right)^{*}-F_{\beta \sigma} F^{\beta \sigma},
$$

where $F_{\beta \sigma}$ is the strength tensor of the Maxwell field. The covariant derivative has the form $\hat{D}_{\beta}=\hat{\nabla}_{\beta}+i e A_{\beta}$, where $e$ is the electric coupling constant, $A_{\beta}$ is the four-potential and $i$ denotes the imaginary unit.

The dark matter Lagrangian is the following:

$$
\hat{\mathcal{L}}_{D M}=-\hat{\widetilde{D}}_{\beta} \chi\left(\hat{\widetilde{D}}^{\beta} \chi\right)^{*}-\frac{1}{4} B_{\beta \sigma} B^{\beta \sigma}-\frac{\alpha_{D M}}{4} B_{\beta \sigma} F^{\beta \sigma}-V\left(|\chi|^{2}\right) .
$$

The covariant derivative is $\hat{\widetilde{D}}_{\beta}=\hat{\nabla}_{\beta}+i \tilde{e} P_{\beta}$, while $P_{\beta}$ is the four-potential of a U(1)gauge field, $\tilde{e}$ is its coupling constant with $\chi$ and $B_{\beta \sigma} \equiv \partial_{\beta} P_{\sigma}-\partial_{\sigma} P_{\beta}$. The scalar field potential is given by $V\left(|\chi|^{2}\right)=\frac{m^{2}}{2}|\chi|^{2}+\frac{\lambda_{D M}}{4}|\chi|^{4}$. The constants $m^{2}$ and $\lambda_{D M}$ are a square of the mass parameter of the scalar field and its quartic self-interaction coupling constant, respectively. The square of the mass parameter can be either positive or negative, when it is negative the scalar field possesses a non-zero vacuum expectation value. On a side note, due to the system of units, which was used in our calculations, it is impossible to compare the parameter $m^{2}$ with the particles masses directly. The perturbativity of the quantized theory requires that $\lambda_{D M}$ is in general smaller than $4 \pi$. In the considered theory it is 
restricted to values not exceeding 0.2 [48]. The parameter $\alpha_{D M}$ stands for the kinetic mixing coupling constant, which controls the strength of the mixing between the photon and the dark photon. Taking into account various experimental scenarios, its upper value is constrained to be not larger than $10^{-3}$ [91].

The searches for the dark photon have been conducted with the use of diverse experimental techniques. The current constraints on its mass and its coupling constant with photon come from a collection of experiments, which are adapted to inspecting their various value ranges. The respective upper limits obtained during the dish antenna experiments are $3.1 \mathrm{eV}$ and $6 \cdot 10^{-12}$ [92]. Studying the supernovae excess cooling covers the $\alpha_{D M}$ values from $10^{-10}$ to $10^{-6}$ and masses within the range $10^{-3}-1 \mathrm{GeV}$ [93]. The beam dump experiments [94, 95], searching for lepton jets in $p p$ collisions [96], investigating heavy neutrino decays [97] and analyses of the distortion of the CMB [98] allowed examining the kinetic mixing parameter from within the range $10^{-7}-10^{-4}$ for the dark photon mass ranges $0.03-0.63 \mathrm{GeV}, 0.4-1.1 \mathrm{GeV}, 1-500 \mathrm{MeV}$ and $10^{-14}-10^{-7} \mathrm{eV}$, respectively. Optical experiments dealt with the dark photon masses between $10^{-5}$ and $10^{-2} \mathrm{eV}$ and the values of the mixing parameter greater than $10^{-7}$ [99]. The upper limit of $\alpha_{D M}$ was established as equal to $1.7 \cdot 10^{-5}$ for the mass range $5-470 \mathrm{MeV}$ in experiments focused on analyzing meson decays [100-102]. Investigating $e^{+} e^{-}$collisions allowed searching the mass range $0.02-10.2 \mathrm{GeV}$ [47], while analyses of the $e^{-}$and $p$ scattering on nuclei focused on $175-550 \mathrm{MeV}$ range [103-105], both with the values of the coupling constant with photon of the order of $10^{-4}-10^{-3}$.

The variation of the action (2.1) with respect to adequate fields, namely phantom scalar $\phi$, Maxwell $A_{\mu}$, complex scalar fields $\psi$ and $\chi$, as well as the $P_{\mu}$ gauge field leads to the following set of equations of motion:

$$
\begin{aligned}
\nabla^{2} \phi+\frac{1}{2 \xi} e^{-2 \phi} F_{\beta \sigma} F^{\beta \sigma} & =0, \\
\nabla_{\mu}\left(e^{-2 \phi} F^{\mu \nu}\right)+\frac{1}{4}\left[i e \psi^{*} D^{\nu} \psi-i e \psi\left(D^{\nu} \psi\right)^{*}\right]+\frac{1}{2} \alpha_{D M} \nabla_{\mu} B^{\mu \nu} & =0, \\
\nabla^{2} \psi+i e A^{\beta}\left(2 \nabla_{\beta}+i e A_{\beta}\right) \psi+i e \nabla_{\beta} A^{\beta} \psi & =0, \\
\nabla^{2} \psi^{*}-i e A^{\beta}\left(2 \nabla_{\beta}-i e A_{\beta}\right) \psi^{*}-i e \nabla_{\beta} A^{\beta} \psi^{*} & =0, \\
\nabla^{2} \chi-\tilde{e}^{2} P_{\beta} P^{\beta} \chi+\frac{1}{2} i \tilde{e}\left[\nabla^{\beta}\left(\chi P_{\beta}\right)+P_{\beta}\left(\nabla^{\beta} \chi\right)\right]-\frac{m^{2}}{2} \chi-\frac{\lambda_{D M}}{2}|\chi|^{2} \chi & =0, \\
\nabla^{2} \chi^{*}-\tilde{e}^{2} P_{\beta} P^{\beta} \chi^{*}-\frac{1}{2} i \tilde{e}\left[\nabla^{\beta}\left(\chi^{*} P_{\beta}\right)+P_{\beta}\left(\nabla^{\beta} \chi^{*}\right)\right]-\frac{m^{2}}{2} \chi^{*}-\frac{\lambda_{D M}}{2}|\chi|^{2} \chi^{*} & =0, \\
\nabla_{\mu} B^{\mu \nu}-2 \tilde{e}^{2} P^{\nu}|\chi|^{2}-i \tilde{e}\left(\chi \nabla^{\nu} \chi^{*}-\chi^{*} \nabla^{\nu} \chi\right)+\frac{1}{2} \alpha_{D M} \nabla_{\mu} F^{\mu \nu} & =0 .
\end{aligned}
$$

During the derivation of the above evolution equations the string frame was converted into the Einstein frame in order to get manageable equations of motion. The metrics in these two frames are related via the conformal transformation

$$
g_{\mu \nu}=e^{-2 \phi} \hat{g}_{\mu \nu}
$$

where $g_{\mu \nu}$ and $\hat{g}_{\mu \nu}$ denote metric tensors in the Einstein and string frames, respectively [106]. The transformation between frames (2.11) preserves causality. Since the inter- 
pretation of the obtained results is based mainly on investigating notions related to causal structures of emerging spacetimes, we do not expect the transformation to influence the ultimate conclusions, which can thus be regarded as physically relevant. From now on, all quantities are written in the Einstein frame.

The issue of choosing an adequate conformal frame to describe physics of a particular system has been so far addressed on numerous occasions in research related primarily to cosmology and particle physics (see, e.g., [107-110]). A general conclusion on the equivalence of conformal frames at the classical level is that although the frames are physically equivalent, the interpretations of results obtained within them may differ [111]. However, as was stated above, this drawback does not refer to our case, as the results analysis is based mainly on describing causal properties of spacetimes.

The most convincing arguments on physical relevance of a particular frame could be obtained by comparing theoretical and experimental data. Some attempts in this respect have been made recently in relation to cosmological observations [111-113]. However, they are not yet complete enough to resolve the issue of the conformal frames equivalence satisfactorily and hence the problem of its experimental confirmation still remains open.

The Einstein equations derived by varying the action (2.1) with respect to gravitational field complement the above set of equations, which describes the examined dynamical system. For studying the evolutions of interest, the double null spherically symmetric line element [114] is selected

$$
d s^{2}=-a(u, v)^{2} d u d v+r^{2}(u, v) d \Omega^{2},
$$

where $u$ and $v$ are retarded and advanced time null coordinates, respectively, and $d \Omega^{2}=$ $d \Theta^{2}+\sin ^{2} \Theta d \Phi^{2}$ is the line element of the unit sphere, where $\Theta$ and $\Phi$ are angular coordinates. Such a coordinate choice determines the spacetime foliation for conducting computations, which is $2+2$ [115]. The double null formalism was successfully employed in dynamical gravitational collapse investigations, e.g., [3-6, 8, 9], because it enables to follow the evolution from approximately past null infinity, through the formation of horizons up to the final central singularity in the case of singular spacetimes.

Regarding spherical symmetry, the only non-vanishing components of the field tensors are $F_{u v}, F_{v u}, B_{u v}$ and $B_{v u}$. Due to the gauge freedom $A_{u} \rightarrow A_{u}+\nabla_{u} \theta^{\prime}$ and $P_{u} \rightarrow P_{u}+\nabla_{u} \theta^{\prime \prime}$, where $\theta^{\prime}=\int A_{v} d v$ and $\theta^{\prime \prime}=\int P_{v} d v$, the only non-zero four-vector components are $A_{u}$ and $P_{u}$. They are functions of retarded and advanced time.

The equation of motion for the phantom scalar field (2.4) in the chosen coordinate system is provided by

$$
r_{, u} \phi_{, v}+r_{, v} \phi_{, u}+r \phi_{, u v}+\frac{1}{\xi} e^{-2 \phi} \frac{Q^{2} a^{2}}{4 r^{3}}=0
$$

where we set

$$
Q=2 \frac{A_{u, v} r^{2}}{a^{2}}
$$

$Q$ is a function of retarded and advanced time, which corresponds to electric charge within a sphere of a radius $r(u, v)$, on a spacelike hypersurface containing the point $(u, v)$. Partial 
derivatives with respect to the null coordinates are marked as ${ }_{, u}$ and ${ }_{, v}$. Concerning the assumed line element (2.12) and the definition of electric charge (2.14), the $v$-component of Maxwell equations (2.5) can be separated into two first-order differential equations. The first one governs the evolution of the only non-zero component of the four-vector of the Maxwell field

$$
A_{u, v}-\frac{Q a^{2}}{2 r^{2}}=0
$$

while the second one describes the dynamical behavior of $Q$. Namely, one has

$$
Q_{, v}-2 \phi_{, v} Q+\frac{i e r^{2}}{4} e^{2 \phi}\left(\psi^{*} \psi_{, v}-\psi \psi_{, v}^{*}\right)+\frac{1}{8} \alpha_{D M} e^{2 \phi} T_{, v}=0,
$$

where we denoted by $T$ the expression

$$
T=\frac{2 r^{2}}{a^{2}} P_{u, v},
$$

being related to the charge associated with the $P_{\mu}$ field within a sphere of a radius $r(u, v)$ on a specific spacelike hypersurface containing $(u, v)$. The relations for the complex scalar field (2.6)-(2.7) imply

$$
\begin{aligned}
r_{, u} \psi_{, v}+r_{, v} \psi_{, u}+r \psi_{, u v}+i e r A_{u} \psi_{, v}+i e r_{, v} A_{u} \psi+\frac{i e Q a^{2}}{4 r} \psi & =0, \\
r_{, u} \psi_{, v}^{*}+r_{, v} \psi_{, u}^{*}+r \psi_{, u v}^{*}-i e r A_{u} \psi_{, v}^{*}-i e r_{, v} A_{u} \psi^{*}-\frac{i e Q a^{2}}{4 r} \psi^{*} & =0 .
\end{aligned}
$$

The equations of the scalar field $\chi$, its complex conjugate $\chi^{*}$ and the related $\mathrm{U}(1)$-gauge field are given by

$$
\begin{aligned}
& r \chi_{, u v}+r_{, u} \chi_{, v}+r_{, v} \chi_{, u}+\frac{1}{4} i \tilde{e} \chi r P_{u, v}+\frac{1}{2} i \tilde{e} r P_{u} \chi_{, v}+\frac{1}{2} i \tilde{e} r_{, v} P_{u} \chi+ \\
& +\frac{1}{8} m^{2} a^{2} r \chi+\frac{\lambda_{D M}}{8} a^{2} r|\chi|^{2} \chi=0, \\
& r \chi_{, u v}^{*}+r_{, u} \chi_{, v}^{*}+r_{, v} \chi_{, u}^{*}-\frac{1}{4} i \tilde{e} \chi^{*} r P_{u, v}-\frac{1}{2} i \tilde{e} r P_{u} \chi_{, v}^{*}-\frac{1}{2} i \tilde{e} r_{, v} P_{u} \chi^{*}+ \\
& +\frac{1}{8} m^{2} a^{2} r \chi^{*}+\frac{\lambda_{D M}}{8} a^{2} r|\chi|^{2} \chi^{*}=0, \\
& T_{, v}-2 i \tilde{e} r^{2}\left(\chi \chi_{, v}^{*}-\chi^{*} \chi_{, v}\right)+\frac{1}{2} \alpha_{D M} Q_{, v}=0, \\
& P_{u, v}-\frac{T a^{2}}{2 r^{2}}=0 .
\end{aligned}
$$

The stress-energy tensor for the considered theory is the following:

$$
\begin{aligned}
T_{\mu \nu}= & 2 \xi \phi_{, \mu} \phi_{, \nu}-g_{\mu \nu} \xi \phi_{, \beta} \phi^{\beta}+e^{-2 \phi}\left(2 F_{\mu \beta} F_{\nu}{ }^{\beta}-\frac{1}{2} g_{\mu \nu} F_{\beta \sigma} F^{\beta \sigma}\right)+ \\
& -\frac{1}{4} g_{\mu \nu} D_{\beta} \psi\left(D^{\beta} \psi\right)^{*}+\frac{1}{4}\left[D_{\mu} \psi\left(D_{\nu} \psi\right)^{*}+\left(D_{\mu} \psi\right)^{*} D_{\nu} \psi\right]+ \\
& +\frac{1}{2}\left[\widetilde{D}_{\mu} \chi\left(\widetilde{D}_{\nu} \chi\right)^{*}+\left(\widetilde{D}_{\mu} \chi\right)^{*} \widetilde{D}_{\nu} \chi\right]+B_{\mu \beta} B_{\nu}{ }^{\beta}+\alpha_{D M} B_{\mu \beta} F_{\nu}{ }^{\beta}+
\end{aligned}
$$




$$
-g_{\mu \nu}\left[\widetilde{D}_{\beta} \chi\left(\widetilde{D}^{\beta} \chi\right)^{*}+\frac{1}{4} B_{\beta \sigma} B^{\beta \sigma}+\frac{\alpha_{D M}}{4} B_{\beta \sigma} F^{\beta \sigma}+V\left(|\chi|^{2}\right)\right] .
$$

Its non-vanishing components calculated in double null coordinates are of the forms

$$
\begin{aligned}
T_{u u}= & 2 \xi \phi_{, u}^{2}+\frac{1}{2}\left[\psi_{, u} \psi_{, u}^{*}+i e A_{u}\left(\psi \psi_{, u}^{*}-\psi^{*} \psi_{, u}\right)+e^{2} A_{u}^{2} \psi \psi^{*}\right]+ \\
& +\chi_{, u} \chi_{, u}^{*}+i \tilde{e} P_{u}\left(\chi \chi_{, u}^{*}-\chi^{*} \chi_{, u}\right)+\tilde{e}^{2} P_{u}^{2} \chi \chi^{*} \\
T_{v v}= & 2 \xi \phi_{, v}^{2}+\frac{1}{2} \psi_{, v} \psi_{, v}^{*}+\chi \chi_{, v} \chi_{, v}^{*} \\
T_{u v}= & e^{-2 \phi} \frac{Q^{2} a^{2}}{2 r^{4}}+\frac{T^{2} a^{2}}{4 r^{4}}+\alpha_{D M} \frac{T Q a^{2}}{4 r^{4}}+\frac{a^{2}}{4}\left(m^{2}|\chi|^{2}+\frac{\lambda_{D M}}{2}|\chi|^{4}\right) \\
T_{\theta \theta}= & 4 \xi \frac{r^{2}}{a^{2}} \phi_{, u} \phi_{, v}+e^{-2 \phi} \frac{Q^{2}}{r^{2}}+\frac{1}{2} \frac{r^{2}}{a^{2}}\left[\psi_{, u} \psi_{, v}^{*}+\psi_{, v} \psi_{, u}^{*}+i e A_{u}\left(\psi \psi_{, v}^{*}-\psi^{*} \psi_{, v}\right)\right]+ \\
& +\frac{T^{2}}{2 r^{2}}+\alpha_{D M} \frac{T Q}{2 r^{2}}+\frac{2 r^{2}}{a^{2}}\left[\chi \chi_{, u} \chi_{, v}^{*}+\chi_{, v} \chi_{, u}^{*}+i \tilde{e} P_{u}\left(\chi \chi_{, v}^{*}-\chi^{*} \chi_{, v}\right)\right]
\end{aligned}
$$

Combining the adequate components of the Einstein tensor resulting from the metric (2.12) and the above stress-energy tensor components, the Einstein equations of the gravitational field are obtained

$$
\begin{aligned}
\frac{2 a_{, u} r_{, u}}{a}-r_{, u u}= & \frac{r}{4}\left[\psi_{, u} \psi_{, u}^{*}+i e A_{u}\left(\psi \psi_{, u}^{*}-\psi^{*} \psi_{, u}\right)+e^{2} A_{u}^{2} \psi \psi^{*}\right]+ \\
& +\xi r \phi_{, u}^{2}+\frac{r}{2}\left[\chi_{, u} \chi_{, u}^{*}+i \tilde{e} P_{u}\left(\chi \chi_{, u}^{*}-\chi^{*} \chi_{, u}\right)+\tilde{e}^{2} P_{u}^{2} \chi \chi^{*}\right] \\
\frac{2 a_{, v} r_{, v}}{a}-r_{, v v}= & \xi r \phi_{v}^{2}+\frac{r}{4} \psi_{, v} \psi_{, v}^{*}+\frac{r}{2} \chi_{, v} \chi_{, v}^{*} \\
\frac{a^{2}}{4 r}+\frac{r_{, u} r_{v}}{r}+r_{, u v}= & e^{-2 \phi} \frac{Q^{2} a^{2}}{4 r^{3}}+ \\
& +\frac{T^{2} a^{2}}{8 r^{3}}+\alpha_{D M} \frac{T Q a^{2}}{8 r^{3}}+\frac{r a^{2}}{4}\left(\frac{m^{2}}{2}|\chi|^{2}+\frac{\lambda_{D M}}{4}|\chi|^{4}\right) \\
\frac{a_{, u} a_{, v}}{a^{2}}-\frac{a_{, u v}}{a}-\frac{r_{, u v}}{r}= & e^{-2 \phi} \frac{Q^{2} a^{2}}{4 r^{4}}+\xi \phi_{, u} \phi_{, v}+\frac{1}{8}\left[\psi_{, u} \psi_{, v}^{*}+\psi_{, u}^{*} \psi_{, v}+i e A_{u}\left(\psi \psi_{v}^{*}-\psi^{*} \psi_{, v}\right)\right]+ \\
& +\frac{T^{2} a^{2}}{8 r^{4}}+\alpha_{D M} \frac{T Q a^{2}}{8 r^{4}}+ \\
& +\frac{1}{2}\left[\chi_{, u} \chi_{, v}^{*}+\chi_{, u}^{*} \chi_{, v}+i \tilde{e} P_{u}\left(\chi \chi_{v}^{*}-\chi^{*} \chi_{, v}\right)\right]
\end{aligned}
$$

They complement the preceding relations in order to obtain the complete set of equations of motion for the examined system, which are (2.13)-(2.32), excluding the relations (2.14), (2.17) and (2.24), which determine the physical quantities $Q, T$ and $T_{\mu \nu}$.

In order to make the solution of the obtained equations of motion attainable, one introduces a set of auxiliary variables

$$
\begin{aligned}
& c=\frac{a_{, u}}{a}, \quad d=\frac{a_{, v}}{a}, \quad f=r_{, u}, \quad g=r_{, v}, \\
& k=\phi, \quad x=\phi_{, u}, \quad y=\phi_{, v}, \\
& s=\psi, \quad p=\psi_{, u}, \quad q=\psi_{, v}, \quad \beta=A_{u}, \\
& h=\chi, \quad w=\chi_{, u}, \quad z=\chi_{, v}, \quad \gamma=P_{u},
\end{aligned}
$$


and the quantities denoted by

$$
\lambda \equiv \frac{a^{2}}{4}+f g, \quad \mu \equiv f q+g p, \quad \kappa \equiv g w+f z,
$$

which make it possible to rewrite the second-order differential equations (2.13), (2.18)(2.21) and (2.29)-(2.32) as first-order ones. Moreover, the real fields $\psi_{1}, \psi_{2}, \chi_{1}$ and $\chi_{2}$ are introduced instead of conjugate fields $\psi, \psi^{*}, \chi$ and $\chi^{*}$ according to $\psi=\psi_{1}+i \psi_{2}$, $\psi^{*}=\psi_{1}-i \psi_{2}, \chi=\chi_{1}+i \chi_{2}$ and $\chi^{*}=\chi_{1}-i \chi_{2}$. These relations result in

$$
\begin{aligned}
s & =s_{1}+i s_{2}, & p & =p_{1}+i p_{2}, & q & =q_{1}+i q_{2}, \\
h & =h_{1}+i h_{2}, & w & =w_{1}+i w_{2}, & z & =z_{1}+i z_{2}, \\
\mu & =\mu_{1}+i \mu_{2}, & \mu_{1} & =f q_{1}+g p_{1}, & \mu_{2} & =f q_{2}+g p_{2}, \\
\kappa & =\kappa_{1}+i \kappa_{2}, & \kappa_{1} & =f z_{1}+g w_{1}, & \kappa_{2} & =f z_{2}+g w_{2} .
\end{aligned}
$$

The final system of equations of motion, which governs the investigated evolution yields

$$
\begin{aligned}
& P 1: a_{, u}=a c \\
& P 2: a_{, v}=a d \\
& P 3: r_{, u}=f \\
& P 4: r_{, v}=g \\
& P 5: s_{1(2), u}=p_{1(2)} \\
& P 6: s_{1(2), v}=q_{1(2)} \\
& P 7: h_{1(2), u}=w_{1(2)} \\
& P 8: h_{1(2), v}=z_{1(2)}, \\
& E 1: f_{, u}=2 c f-r x^{2}-\frac{r}{4}\left[p_{1}^{2}+p_{2}^{2}+2 e \beta\left(s_{1} p_{2}-s_{2} p_{1}\right)+e^{2} \beta^{2}\left(s_{1}^{2}+s_{2}^{2}\right)\right]+ \\
&-\frac{1}{2} r\left[w_{1}^{2}+w_{2}^{2}+2 \tilde{e} \gamma\left(h_{1} w_{2}-h_{2} w_{1}\right)+\tilde{e}^{2} \gamma^{2}\left(h_{1}^{2}+h_{2}^{2}\right)\right] \\
& E 2: g_{, v}=2 d g-r y^{2}-\frac{r}{4}\left(q_{1}^{2}+q_{2}^{2}\right)-\frac{r}{2}\left(z_{1}^{2}+z_{2}^{2}\right) \\
& E 3: g_{, u}=f_{, v}=-\frac{\lambda}{r}+e^{-2 k} \frac{Q^{2} a^{2}}{4 r^{3}}+\frac{T^{2} a^{2}}{8 r^{3}}+\alpha_{D M} \frac{T Q a^{2}}{8 r^{3}}+ \\
& \quad+\frac{r a^{2}}{4}\left[\frac{m^{2}}{2}\left(h_{1}^{2}+h_{2}^{2}\right)+\frac{\lambda_{D M}}{4}\left(h_{1}^{2}+h_{2}^{2}\right)^{2}\right] \\
& E 4: d_{, u}=c_{, v}=\frac{\lambda}{r^{2}}-x y-\frac{1}{4}\left[p_{1} q_{1}+p_{2} q_{2}+e \beta\left(s_{1} q_{2}-s_{2} q_{1}\right)\right]-e^{-2 k} \frac{Q^{2} a^{2}}{2 r^{4}}+ \\
& \quad-\frac{T^{2} a^{2}}{8 r^{4}}-\alpha_{D M} \frac{T Q a^{2}}{8 r^{4}}-w_{1} z_{1}-w_{2} z_{2}+\tilde{e} \gamma\left(h_{2} z_{1}-h_{1} z_{2}\right) \\
& S_{(I m)}: q_{2, u}=p_{2, v}=-\frac{\mu_{2}}{r}-e \beta q_{1}-e s_{1} \beta \frac{g}{r}-e s_{1} \frac{Q a^{2}}{4 r^{2}} \\
& M 1: \beta_{, v}=\frac{Q a^{2}}{2 r^{2}}, \\
& S_{1, u}=p_{1, v}=-\frac{\mu_{1}}{r}+e \beta q_{2}+e s_{2} \beta \frac{g}{r}+e s_{2} \\
& 4 r^{2}
\end{aligned}
$$




$$
\begin{array}{cl}
M 2: & Q_{, v}=\left[2 y Q+\frac{1}{2} e r^{2} e^{2 k}\left(s_{1} q_{2}-s_{2} q_{1}\right)+\right. \\
+ & \left.\frac{\alpha_{D M}}{4} \tilde{e} r^{2} e^{2 k}\left(z_{1} h_{2}-z_{2} h_{1}\right)\right]\left(1-e^{2 k} \frac{\alpha_{D M}^{2}}{16}\right)^{-1}, \\
H_{(R e)}: & z_{1, u}=w_{1, v}=-\frac{\kappa_{1}}{r}+\tilde{e} h_{2} \frac{T a^{2}}{8 r^{2}}+\frac{1}{2} \tilde{e} z_{2} \gamma+\frac{1}{2} \tilde{e} h_{2} \gamma \frac{g}{r}+ \\
& -\frac{1}{8} a^{2} h_{1}\left[m^{2}+\lambda_{D M}\left(h_{1}^{2}+h_{2}^{2}\right)\right] \\
H_{(I m)}: & z_{2, u}=w_{2, v}=-\frac{\kappa_{2}}{r}-\tilde{e} h_{1} \frac{T a^{2}}{8 r^{2}}-\frac{1}{2} \tilde{e} z_{1} \gamma-\frac{1}{2} \tilde{e} h_{1} \gamma \frac{g}{r}+ \\
& -\frac{1}{8} a^{2} h_{2}\left[m^{2}+\lambda_{D M}\left(h_{1}^{2}+h_{2}^{2}\right)\right] \\
C 1: & \gamma_{, v}=\frac{T a^{2}}{2 r^{2}}, \\
C 2: & T_{, v}=2 \tilde{e} r^{2}\left(h_{1} z_{2}-h_{2} z_{1}\right)-\frac{\alpha_{D M}}{2}\left[2 y Q+\frac{1}{2} e r^{2} e^{2 k}\left(s_{1} q_{2}-s_{2} q_{1}\right)+\right. \\
& \left.+\frac{\alpha_{D M}}{4} \tilde{e} r^{2} e^{2 k}\left(z_{1} h_{2}-z_{2} h_{1}\right)\right]\left(1-e^{2 k} \frac{\alpha_{D M}^{2}}{16}\right)^{-1} \cdot
\end{array}
$$

\section{Details of computer simulations and results analysis}

Because of its complexity, the system of the obtained differential equations (2.36)-(2.55) needs to be solved numerically. The details of the numerical code and performed tests are presented in appendix A.

The evolution equations were solved in the region of the $(v u)$-plane, which is shown on the background of a dynamical Reissner-Nordström spacetime [6] in figure 1. In all conducted simulations it was confined to $0 \leqslant v \leqslant 7.5$ and $0 \leqslant u \leqslant 7.5$. The only arbitrary input data of the computations were profiles of the evolving fields, posed on the initial null hypersurface denoted as $u=0$. The initial profile of the phantom scalar field was Gaussian

$$
\phi=\tilde{p}_{k} \cdot v^{2} \cdot e^{-\left(\frac{v-c_{1}}{c_{2}}\right)^{2}}
$$

while the complex fields were modeled by the trigonometric profile of the following form:

$$
\psi(\text { or } \chi)=\tilde{p}_{s}\left(\text { or } \tilde{p}_{h}\right) \cdot \sin ^{2}\left(\pi \frac{v}{v_{f}}\right) \cdot\left[\cos \left(\pi \frac{2 v}{v_{f}}\right)+i \cos \left(\pi \frac{2 v}{v_{f}}+\delta\right)\right] .
$$

The above profiles were selected so that they described the behavior of real and complex scalar fields properly $[5,6,116,117]$. The profiles were treated as one-parameter families with amplitudes $\tilde{p}_{k}, \tilde{p}_{s}$ and $\tilde{p}_{h}$ as free family parameters. The amplitudes are indicators of the strength of the gravitational self-interaction of the particular field [118]. The existence of a specific matter type in the examined system was guaranteed by the non-zero value of the respective amplitude. Three systems were investigated, i.e., 


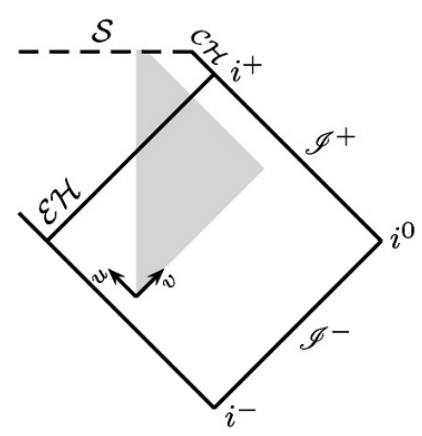

Figure 1. The computational domain (marked gray) on the background of the Carter-Penrose diagram of the dynamical Reissner-Nordström spacetime. The central singularity along $r=0$, the event and Cauchy horizons are denoted as $\mathcal{S}, \mathcal{E H}$ and $\mathcal{C H}$, respectively. $\mathscr{I}^{ \pm}$and $i^{ \pm}$are null and timelike infinities, while $i^{0}$ is a spacelike infinity.

- electrically charged scalar field - dark energy $(S F-D E)$ with $\tilde{p}_{s} \neq 0, \tilde{p}_{k} \neq 0$ and $\tilde{p}_{h}=0$,

- electrically charged scalar field - dark matter $(S F-D M)$ with $\tilde{p}_{s} \neq 0, \tilde{p}_{k}=0$ and $\tilde{p}_{h} \neq 0$,

- electrically charged scalar field — dark energy - dark matter $(S F-D E-D M)$ with $\tilde{p}_{s} \neq 0, \tilde{p}_{k} \neq 0$ and $\tilde{p}_{h} \neq 0$.

The remaining constants were arbitrarily chosen and invariable during computations. To be precise, we set $c_{1}=1.3, c_{2}=0.21$ and the parameter determining the amount of initial charge $\delta=\frac{\pi}{2}$. The maximum value of advanced time was $v_{f}=7.5$. The choice of initial conditions is representative for the conducted evolutions, because their outcomes are independent of the types of profiles provided that they are regular, i.e., they result in a regular spacetime slice at the initial null hypersurface. This condition is fulfilled by the selected profiles (3.1) and (3.2).

Provided that the value of the electric coupling constant is non-zero, it does not affect the results of the collapse [14], which was confirmed for the investigated cases. For this reason, it was set as equal to $e=0.5$ in all evolutions. A similar property was observed for the coupling constant $\tilde{e}$, so it was also kept constant and equal to 0.5 during simulations. The cases of vanishing $e$ and $\tilde{e}$ were not examined, because they refer to uncharged complex scalar fields, whose behavior during the collapse is beyond the scope of the current research.

The dynamical spacetime structures resulting from the considered evolutions will be presented on Penrose diagrams. These diagrams contain contours of $r=$ const. lines plotted in the $(v u)$-plane. The outermost thick line refers to $r=0$, which is non-singular when coinciding with the $u=v$ line and singular in the remaining part. The lines indicating the vanishing expansion

$$
\theta_{i} \equiv \frac{2}{r} r_{, i}
$$

in the respective null directions $i=u, v$ will be also presented on the diagrams and their role in a spacetime will be commented in each case separately. They will be denoted 


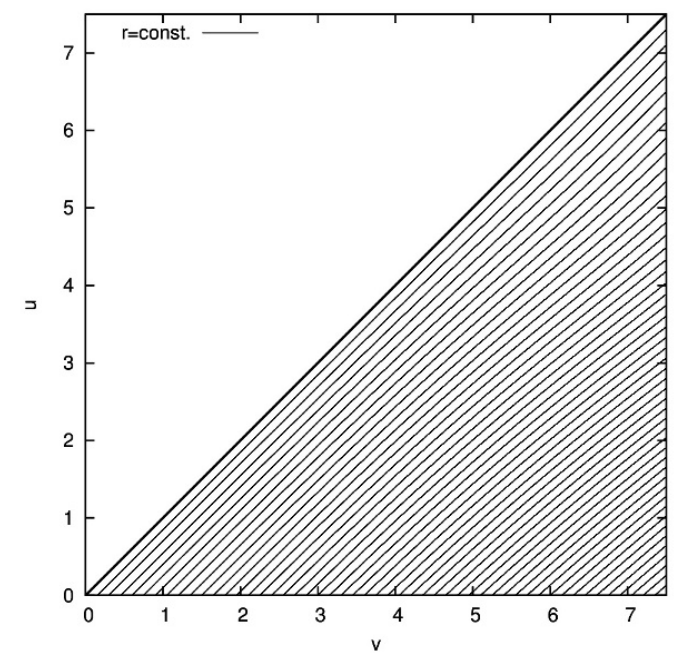

(a)

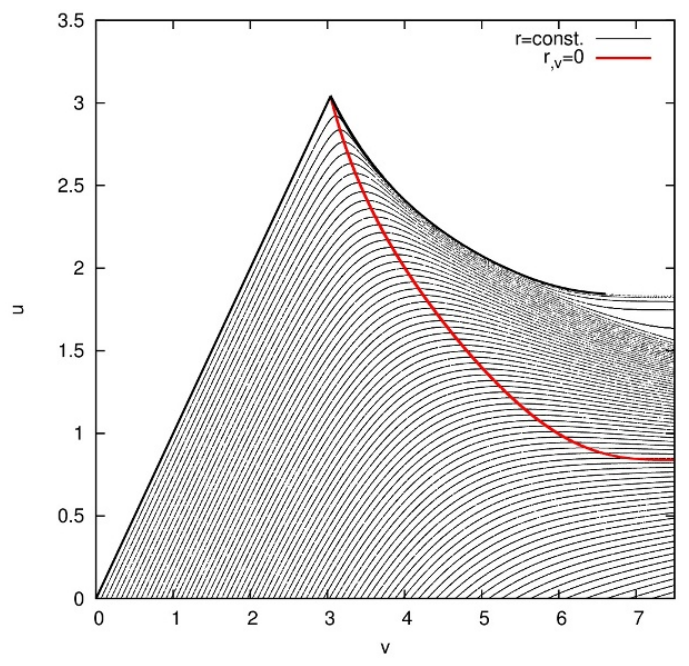

(b)

Figure 2. (color online) Spacetime structures emerging from the collapse of an electrically charged scalar field of the amplitude (a) $\tilde{p}_{s}=0.2$ and (b) $\tilde{p}_{s}=0.6$.

as $r_{, v}=0$ and $r_{, u}=0$ and marked on the diagrams as red and blue solid lines, respectively. The presentation of Penrose diagrams will be limited to spacetime regions, which are crucial for the conducted results analysis.

The aim of the paper is to present the influence of the dark sector on the collapse of an electrically charged scalar field. In figure 2 we present the Penrose diagrams of spacetimes formed during the gravitational evolution of the field without any additional factors as a reference for further analyses. For the initial amplitude value $\tilde{p}_{s}=0.2$ the emerging curved spacetime is non-singular, because the evolving field disperses towards infinity (figure 2a). The process with $\tilde{p}_{s}=0.6$ results in a dynamical Reissner-Nordström spacetime [6], shown in figure $2 \mathrm{~b}$.

The obtained Reissner-Nordström spacetime consists of two parts. The first one covers the dynamical region of spacetime and spreads up to about $v=7.05$. The second one refers to $v>7.05$ and is non-dynamical. This distinction is based on the analysis of the behavior of the apparent horizon, which lies along the $r_{, v}=0$ line. It changes its position along $u$ in the dynamical region and becomes null as $v \rightarrow \infty$. Its location there, along $u=$ 0.84, determines the event horizon location in the spacetime [119]. The Cauchy horizon is situated at the future null infinity, i.e., at $v=\infty$. It is invisible within the covered spacetime domain, but its existence is confirmed by the fact that the $r=$ const. lines tend towards constant $u$ with increasing advanced time. The line $r=0$ is non-singular up to $v=3.04$ and becomes a spacelike singularity for bigger values of advanced time.

One of physical quantities, which plays a significant role during the interpretation of the results is the quasi-local Hawking mass [120]. Its value calculated for the spherically 
symmetric spacetime with two coupled gauge fields $A_{\mu}$ and $P_{\mu}$ is provided by

$$
m(u, v)=\frac{r}{2}\left(1+\frac{4 f g}{a^{2}}+\frac{2 Q^{2}+T^{2}+\alpha_{D M} T Q}{r^{2}}\right) .
$$

It describes the mass contained within a sphere of a radius $r(u, v)$ on a spacelike hypersurface containing the point $(u, v)$. The mass of a particular object is a value of the above expression at the event horizon in the non-dynamical region of the emerging spacetime, i.e., for $v=v_{f}$.

\section{Electrically charged scalar field collapse with dark energy}

An interpretation of the dark energy impact on the examined collapse was made on the basis of two distinct sets of solutions describing the outcomes of the process. The first one corresponds to the varying amplitude of the phantom field $\tilde{p}_{k}$ and the constant amplitude of the electrically charged scalar field $\tilde{p}_{s}=0.6$. The other set was obtained during simulations with the constant phantom scalar field amplitude $\tilde{p}_{k}=0.1$ and the varying charged scalar field amplitude $\tilde{p}_{s}$. Such an approach allows to investigate the influence of the gravitational self-interaction strength of a particular field on the studied process. The evolutions conducted for the selected values of constant amplitudes, when the accompanying field is absent, lead to dynamical spacetimes of Reissner-Nordström and Schwarzschild types, respectively [16].

\subsection{Dynamical emergence of wormhole-like structures and naked singularities}

Dynamical spacetimes resulting from the collapse proceeding for the constant electrically charged scalar field amplitude and varying $\tilde{p}_{k}$ are presented in figure 3 . The emerging spacetimes are singular for small values of $\tilde{p}_{k}$ and become non-singular for its bigger values. This results from the fact that the phantom field contributes negatively to the overall energy of the system. For small values of the amplitude $\tilde{p}_{k}$, up to 0.22 , the spacetimes are singular with both $r_{, u}=0$ and $r_{, v}=0$ lines of zero expansion visible in the domain of integration. For values of the altering amplitude not exceeding 0.185 there are two branches of the $r_{, v}=$ 0 line. The outer one surrounds the singular part of $r=0$ (for explanation, see section 3 ) and it becomes null as $v \rightarrow \infty$. The second branch of the line $r_{, v}=0$ lies inside the first one and its beginning coincides with the end of the singular spacelike $r=0$ line. The line of $r_{, u}=0$ is situated beyond the second branch of $r_{, v}=0$ and begins at the same point. For small values of the parameter $\tilde{p}_{k}$ these two lines practically coincide.

The structure described above, with two branches of $r_{, v}=0$ lines and the line $r_{, u}=0$ beyond them, is a dynamical wormhole. The strong flare-out condition $\theta_{i, i}>0$ with $i=u, v[121]$ is fulfilled along the $r_{, u}=0$ line and the inner branch of $r_{, v}=0$. This fact proves that they are wormhole throats. Each of the throats is related to one travel direction. The outgoing throat is situated along $r_{, u}=0$ and the ingoing one along $r_{, v}=0$. The strong flare-out condition is not satisfied along the outer branch of the line $r_{, v}=0$, because the relation $\theta_{v, v}<0$ holds there. This confirms that the branch is not a throat, 


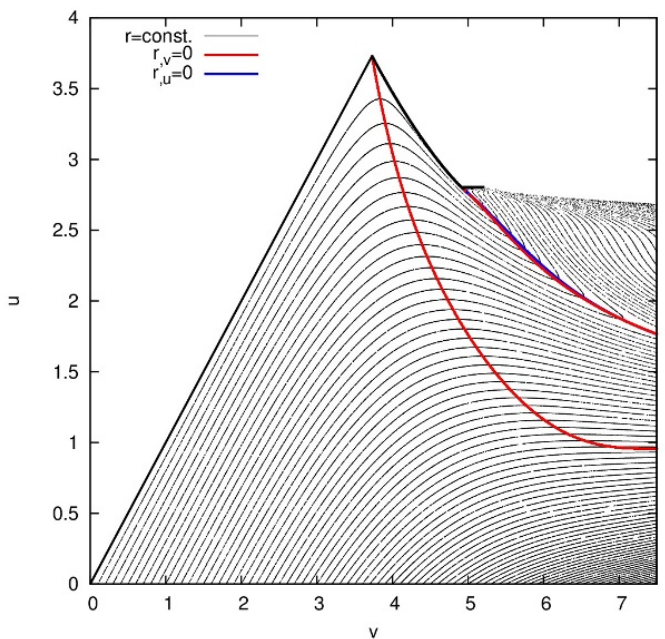

(a)

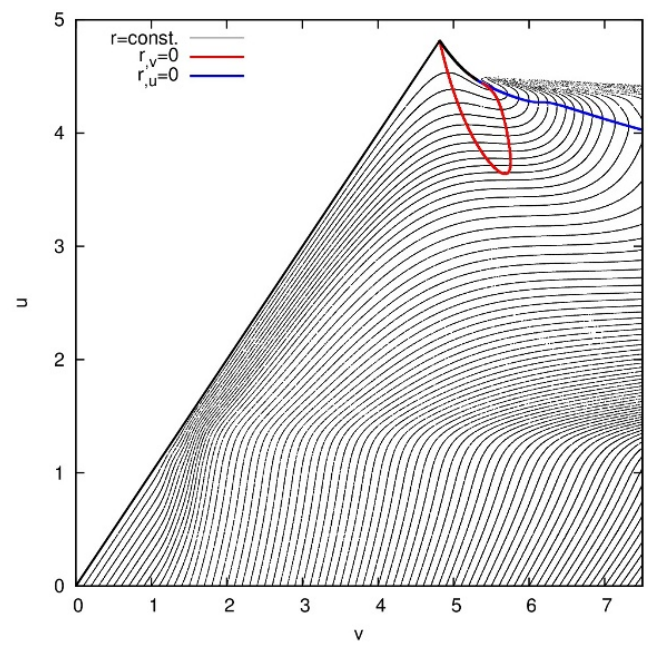

(c)

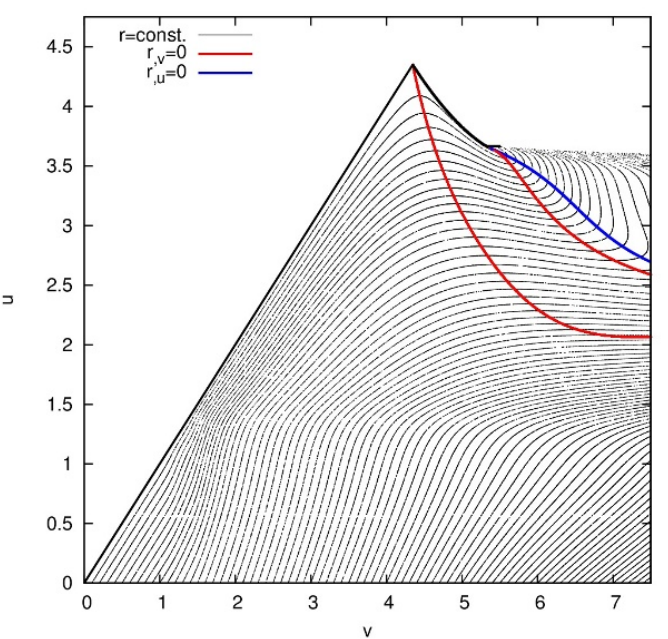

(b)

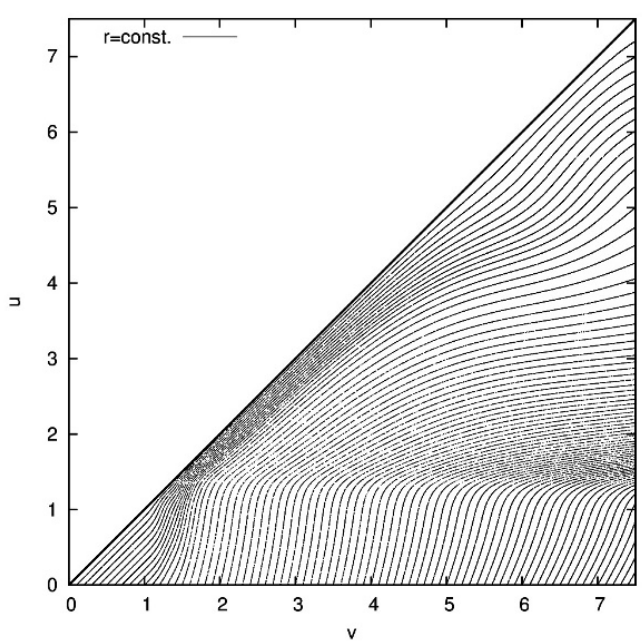

(d)

Figure 3. (color online) Penrose diagrams of spacetimes emerging from the $S F-D E$ evolution with the varying phantom scalar field amplitude. The family parameter for the electrically charged scalar field $\tilde{p}_{s}=0.6$, while for the phantom scalar field $\tilde{p}_{k}$ is equal to (a) 0.01 , (b) 0.15 , (c) 0.2 and (d) 0.25 .

but an apparent horizon in the spacetime, whose null course in the region $v \rightarrow \infty$ indicates the location of an event horizon.

For values of the amplitude $\tilde{p}_{k}$ larger than 0.185 , but not exceeding 0.22 , the two branches of the line $r_{, v}=0$ form a loop, whose ends are joined to the ending points of the singular part of the $r=0$ line. The line $r_{, u}=0$ remains outside the loop. Such a structure corresponds to a naked singularity in the spacetime [9]. For values of the scalar field amplitude $\tilde{p}_{k}$ larger than 0.22 , the spacetime is non-singular.

The second set of solutions involves spacetimes emerging from a collapse when the amplitude of the phantom scalar field is constant and the amplitude of the electrically charged 


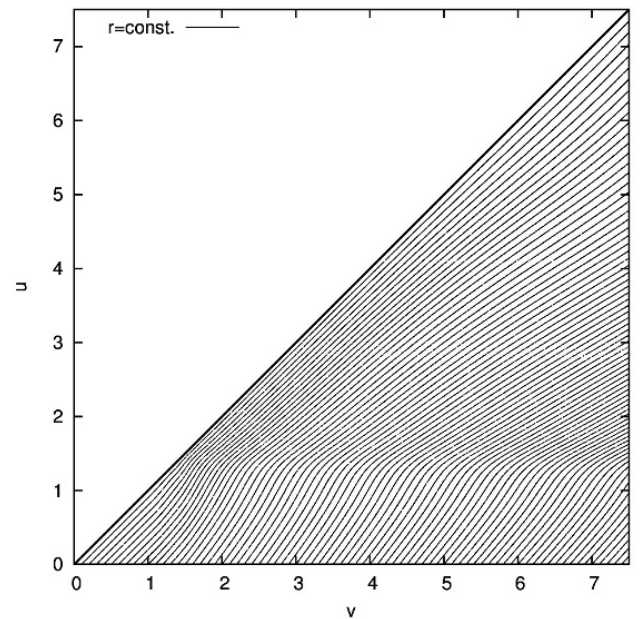

(a)

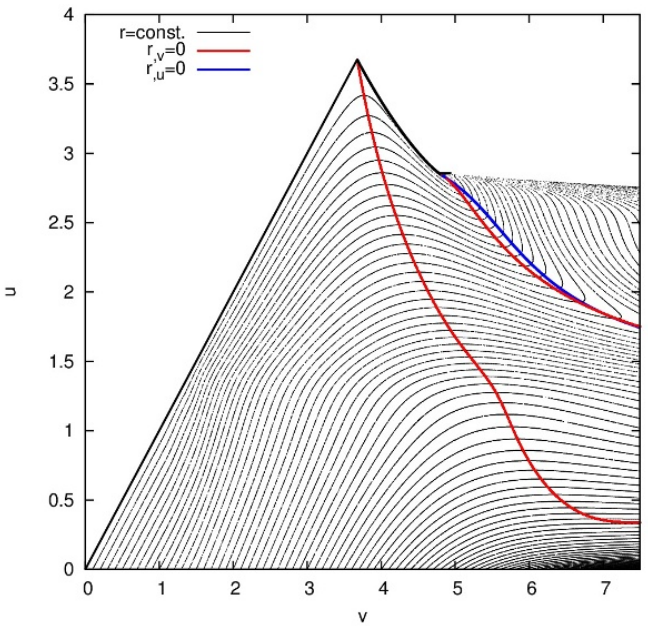

(c)

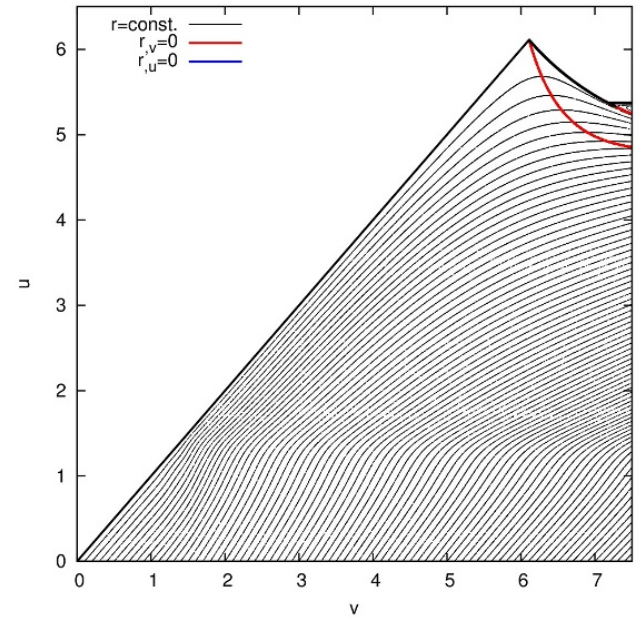

(b)

Figure 4. (color online) Penrose diagrams of spacetimes emerging from the $S F-D E$ evolution with the varying electrically charged scalar field amplitude. The family parameter of the phantom scalar field $\tilde{p}_{k}=0.1$, while $\tilde{p}_{s}$ is equal to (a) 0.3 , (b) 0.375 and (c) 0.7 .

scalar field changes. In figure 4 the structures of spacetimes which stem from the collapse are depicted. As in the previous set of solutions, the wormhole structures are observed. But unlike the preceding case, the spacetime is non-singular for small values of the amplitude $\tilde{p}_{s}$, not exceeding 0.35 , and contains a wormhole for its bigger values. The wormhole structures are similar to these presented above, that is there are two branches of the $r_{, v}=0$ line, the outer and the inner one, and the line $r_{, u}=0$ located within them. The outer branch of the line $r_{, v}=0$ settles along a constant value of $u$-coordinate for $v \rightarrow \infty$ indicating the location of an event horizon. Contrary to the previous case, no naked singularities were observed in the formed dynamical spacetimes. 


\subsection{Vicinity of wormhole throats}

As was explained in the previous section, due to the behavior of the expansion (3.3) and its adequate derivatives, the ingoing and outgoing wormhole throats are located along the inner branch of $r_{, v}=0$ and along the $r_{, u}=0$ line, respectively. As was anticipated and confirmed for eternal wormholes, the violation of the null energy condition (NEC)

$$
T_{\mu \nu} n^{\mu} n^{\nu} \geqslant 0
$$

where $n^{\mu}$ denotes a null vector, is crucial for their existence [121, 122].

In spherical symmetry, taking into account the line element (2.12), the stress-energy tensor can be written in the general form

$$
T_{\mu \nu} d x^{\mu} d x^{\nu}=T_{u u} d u^{2}+T_{v v} d v^{2}+2 T_{u v} d u d v+T_{k k} r^{2} d \Omega^{2},
$$

where $T_{u u}, T_{v v}, T_{u v}$ and $T_{k k}$ are its components, while $k=\Theta, \Phi$. The matter described by the above stress-energy tensor fulfills the null energy condition when diagonal components of (4.2) corresponding to the coordinates $u$ and $v$ are non-negative [123], i.e.,

$$
T_{u u} \geqslant 0 \text { and } T_{v v} \geqslant 0 .
$$

The non-zero components of the stress-energy tensor (2.24) were calculated for the wormhole spacetimes in order to examine the fulfillment of the NEC in the dynamical case. Figure 5 presents the components (2.25)-(2.28) for the $S F$ - $D E$ collapse with the amplitudes of the electrically charged field and the phantom field equal to $\tilde{p}_{s}=0.6, \tilde{p}_{k}=0.15$ and $\tilde{p}_{s}=$ $0.7, \tilde{p}_{k}=0.1$ (the respective spacetime structures were presented in figures $3 \mathrm{~b}$ and $4 \mathrm{c}$ ).

In the vicinity of wormhole throats the violation of the null energy condition is observed, because the requirement (4.3) is not satisfied. At the same time, this condition is fulfilled nearby the singular part of $r=0$. The violation of NEC is thus a factor, which determines the existence of wormhole throats also in the dynamical case. Another area, in which the null energy condition is violated, is the vicinity of null lines $v \approx 1.3$ and $u \approx 1.3$ in both investigated wormhole spacetimes. This region is related to the evolution of the phantom scalar field, during which it neither falls into the central singularity nor evolves through the wormhole, but escapes to null infinity as $v \rightarrow \infty$.

Figures 6 and 7 depict the modulus of the electrically charged complex scalar field and the phantom scalar field in the $(v u)$-plane for the same evolutions as in figure 5. The modulus of the electrically charged scalar field increases with retarded time uniformly for all values of the $v$-coordinate. It is hard to distinguish any area, within which the field accumulates and the biggest values are one order of magnitude larger than the smallest ones. The value of the function related to the phantom field is considerably higher in the region nearby wormhole throats, where it is four orders of magnitude bigger than in the surrounding spacetime region. It can be interpreted as a phenomenon of stabilization of the wormhole structure by phantom matter [70]. The value of the phantom scalar field function is also significantly bigger along the hypersurfaces $v \approx 1.3$ and then $u \approx 1.3$ indicating the course of the dispersive evolution of the field, which was also noticed during the above interpretation of the stress-energy tensor components. 

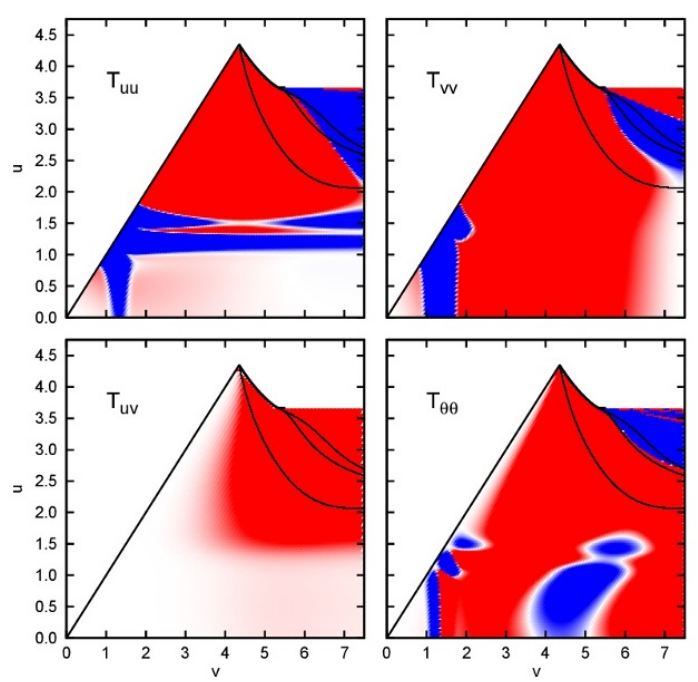

(a)

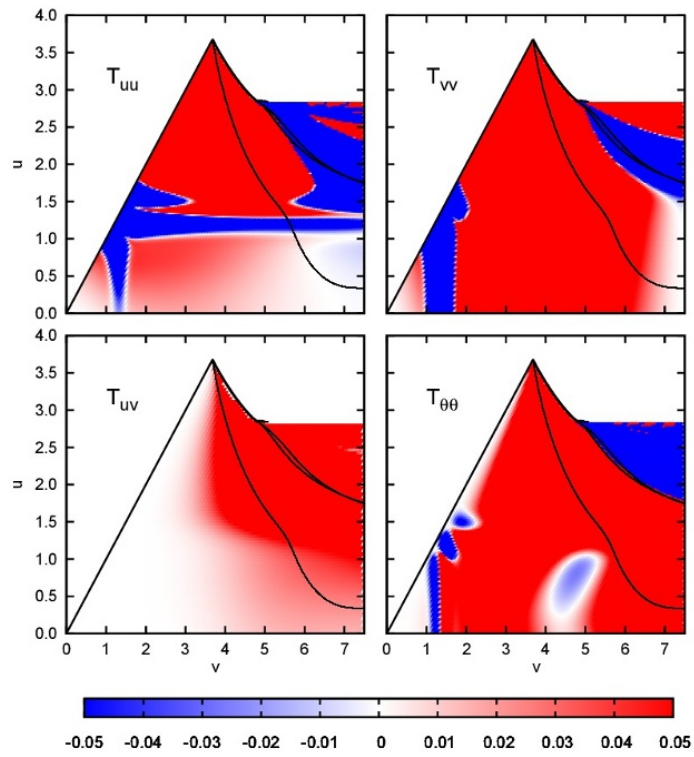

(b)

Figure 5. (color online) The stress-energy tensor components (2.25)-(2.28) in the (vu)-plane for evolutions carried out for electrically charged scalar field and phantom scalar field amplitudes set as (a) $\tilde{p}_{s}=0.6$ and $\tilde{p}_{k}=0.15$, (b) $\tilde{p}_{s}=0.7$ and $\tilde{p}_{k}=0.1$.

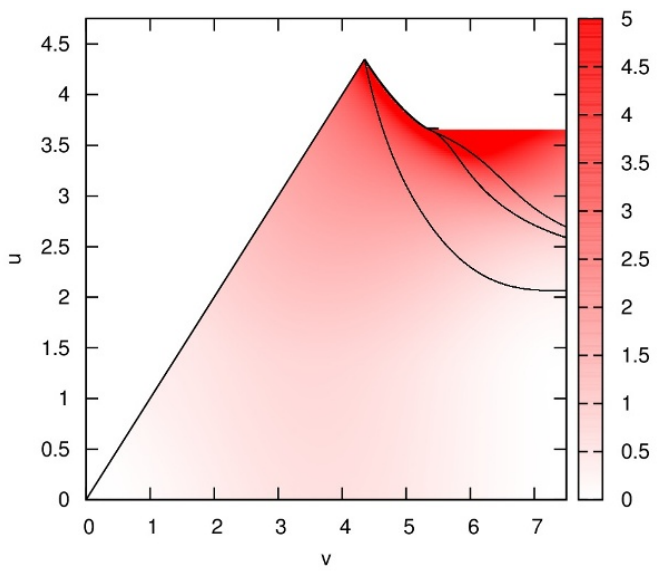

(a)

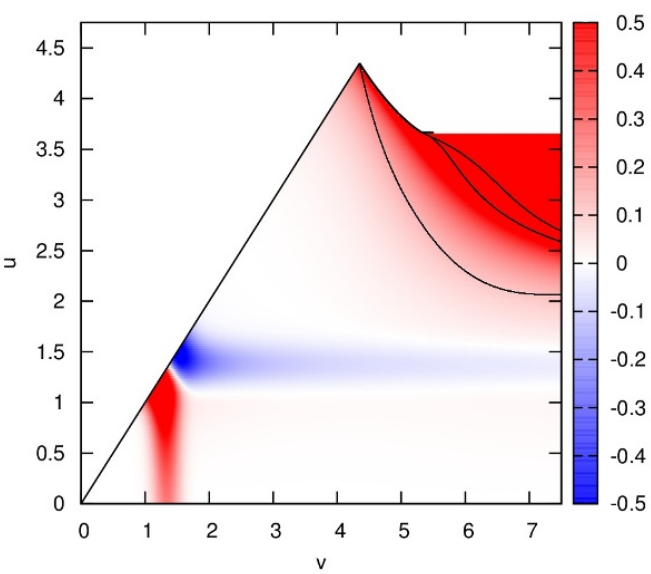

(b)

Figure 6. (color online) The $(v u$ )-distribution of (a) the modulus of the electrically charged scalar field, $|\psi|$, and (b) the phantom scalar field, $\phi$, for the $S F-D E$ evolution characterized by initial field amplitudes $\tilde{p}_{s}=0.6$ and $\tilde{p}_{k}=0.15$. 


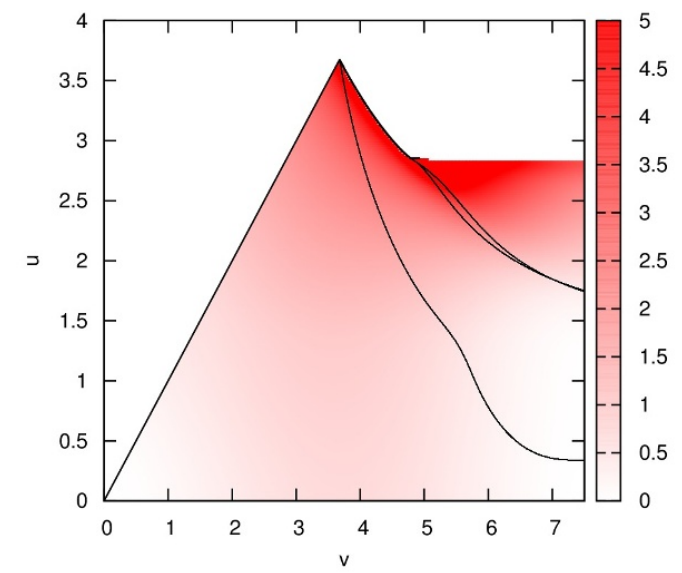

(a)

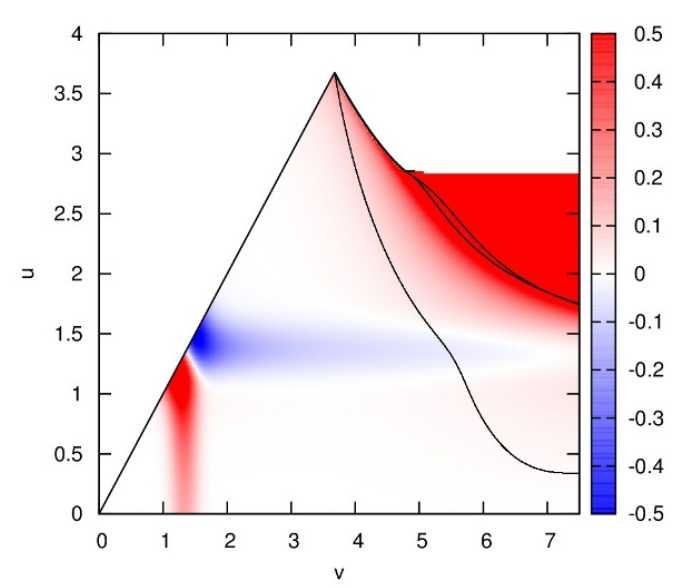

(b)

Figure 7. (color online) The (vu)-distribution of (a) $|\psi|$ and (b) $\phi$ for the $S F-D E$ evolution characterized by initial field amplitudes $\tilde{p}_{s}=0.7$ and $\tilde{p}_{k}=0.1$.

\subsection{Black hole-wormhole duality}

A possibility of a two-way interconversion between black holes and wormholes was discussed in [124-128]. It was stated that a black hole may turn into a wormhole when irradiated by matter with negative energy. On the other hand, a wormhole stabilized by such exotic matter shall turn into a black hole when the matter source disappears.

Dynamical wormhole spacetimes obtained in our computations contain spacelike singularities for large values of retarded time and the existence of event horizons is due to these late- $u$ singularities. A singularity exists during the dynamical stage of the collapse and bifurcates into two wormhole throats when the spacetime tends towards its stationary phase at $v \rightarrow \infty$. Such a course of the investigated process suggests that a black hole, which emerges in the preliminary stage of the evolution, finally converts into a wormhole. The existence of a singularity at large values of retarded time is strictly connected with the fact that the null energy condition is fulfilled in the area (figure 5), what is in turn a result of a considerably smaller value of the phantom field function in this region (figures $6 \mathrm{~b}$ and $7 \mathrm{~b}$ ). The reason for the black hole-wormhole conversion is a substantial increase of its values in the particular area, which in consequence turns into a tunnel outlined by wormhole throats.

\subsection{Properties of wormholes}

The issue whether and in what manner do the properties of the obtained spacetimes differ when one of the field amplitudes is constant and the value of the other amplitude changes was also addressed. The ranges of varying amplitudes were chosen in such a way that in the case of the constant electrically charged scalar field amplitude the biggest $\tilde{p}_{k}$ is approximately the last one not leading to the formation of a naked singularity. In the case of a constant phantom scalar field amplitude the smallest $\tilde{p}_{s}$ refers to the first dynamical wormhole spacetime which appears instead of a non-singular spacetime. 


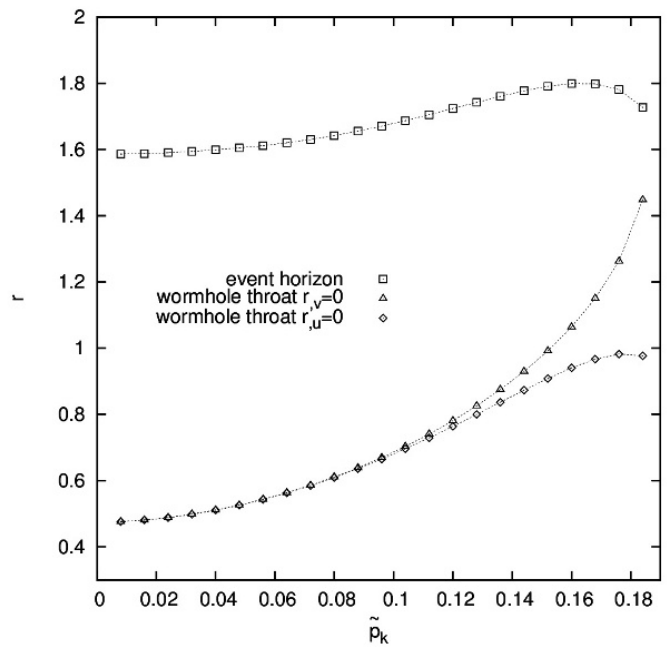

(a)

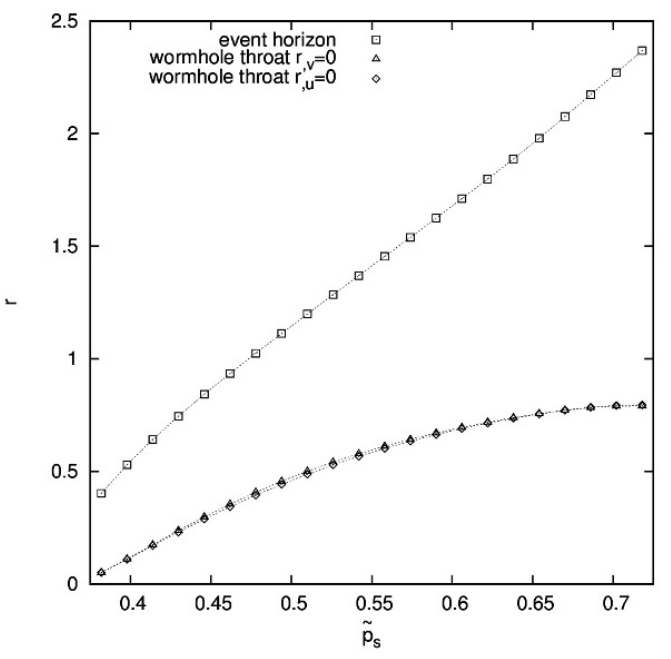

(b)

Figure 8. The radii of event horizons and wormhole throats at the final value of the $v$-coordinate as functions of (a) the phantom scalar field amplitude $\tilde{p}_{k}$ and (b) the electrically charged scalar field amplitude $\tilde{p}_{s}$ for the $S F-D E$ collapse. The constant free family parameters for the particular evolutions were chosen to be equal to $\tilde{p}_{s}=0.6$ and $\tilde{p}_{k}=0.1$, respectively.

In figure 8 the radii of event horizons and wormhole throats at the final value of the $v$ coordinate were plotted as functions of the altering field amplitudes. The chosen value of advanced time $v_{f}$ corresponds to the well-established stationary phase of the evolution due to the fact that in all considered cases the apparent horizon $r_{, v}=0$ is a null hypersurface there. The common feature of both sets of solutions is that the radii of event horizons as well as wormhole throats increase when the varying amplitude increases. The radii of event horizons for the biggest values of $\tilde{p}_{k}$ are the only exception (figure 8a). In this region two branches of the line $r_{, v}=0$ approach each other, which for values of $\tilde{p}_{k}$ exceeding 0.185 results in their connection and naked singularity formation. Such a tendency is not visible for the other set of solutions with the varying amplitude $\tilde{p}_{s}$ (figure $8 \mathrm{~b}$ ), hence a naked singularity does not form. The changes of radii of event horizons are considerably more noticeable in the case of a varying amplitude of the electrically charged scalar field. On the contrary, the radii of wormhole throats vary more significantly when the phantom scalar field amplitude alters. The radii of both wormhole throats are identical for all values of the varying $\tilde{p}_{s}$. For altering $\tilde{p}_{k}$ they are equal up to some point and than they begin to differ more and more significantly.

Figure 9 depicts the $u$-locations of event horizons, wormhole throats at the final value of advanced time and the points, where the line $r=0$ becomes singular (singularity origins) for both sets of solutions. When the phantom scalar field amplitude increases, the $u$ locations of all the mentioned features increase (figure 9a), while in the other case the opposite tendency is observed (figure $9 \mathrm{~b}$ ). Similarly to the radii, the $u$-locations of wormhole throats are exactly the same in the case of altering $\tilde{p}_{s}$ and they bifurcate at some point when $\tilde{p}_{k}$ increases. It is connected with the forthcoming naked singularity formation. 


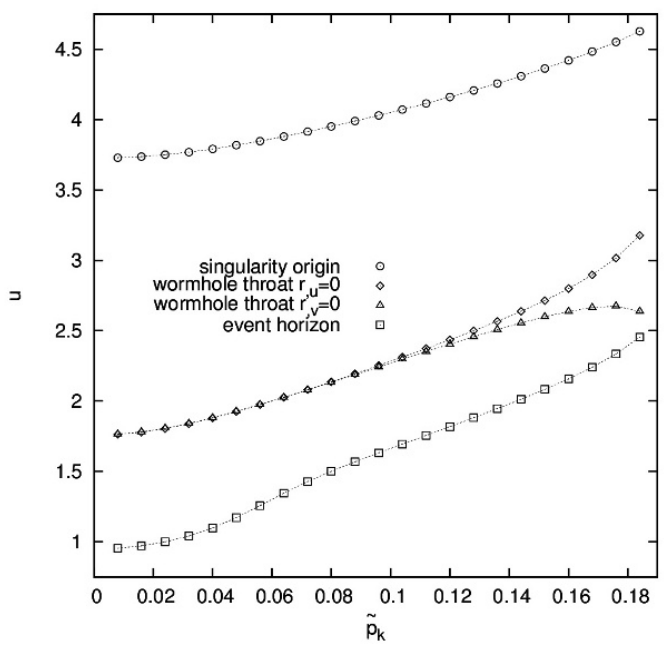

(a)

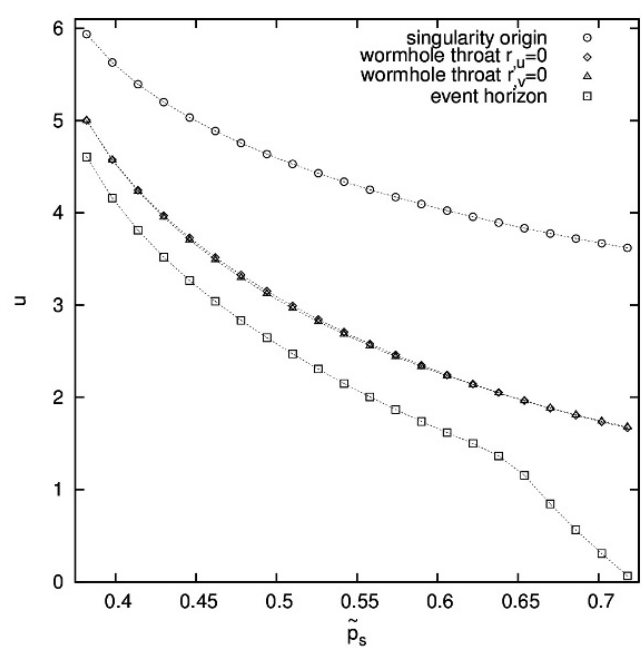

(b)

Figure 9. The $u$-locations of event horizons, wormhole throats at the final value of the $v$-coordinate and the points, where the line $r=0$ becomes singular, as functions of (a) $\tilde{p}_{k}$ and (b) $\tilde{p}_{s}$ for the $S F$ $D E$ collapse. The respective constant free family parameters are the same as in figure 8 .

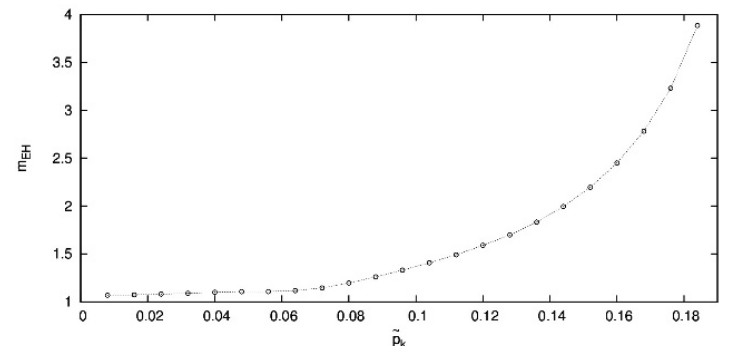

(a)

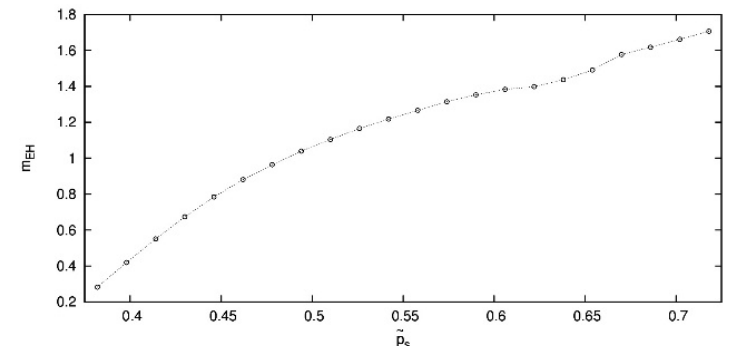

(b)

Figure 10. The wormhole masses, $m_{E H}$, as functions of (a) $\tilde{p}_{k}$ and (b) $\tilde{p}_{s}$ for the $S F-D E$ collapse. The respective constant free family parameters are the same as in figure 8 .

The changes in $u$-locations are considerably bigger when the phantom scalar field amplitude is constant and the amplitude of the electrically charged scalar field varies, in comparison to the other set of solutions.

The masses of wormholes versus the phantom scalar field and electrically charged scalar field amplitudes are presented in figure 10. In both cases the mass increases when the amplitude raises.

\section{Influence of dark matter on electrically charged scalar field evolution}

During examining the role of dark matter in the studied collapse the values of parameters, which characterize the considered dark matter model, i.e., $\alpha_{D M}, m^{2}$ and $\lambda_{D M}$, were varied within the permissible ranges outlined in section 2 . It turned out that $\alpha_{D M}$ 


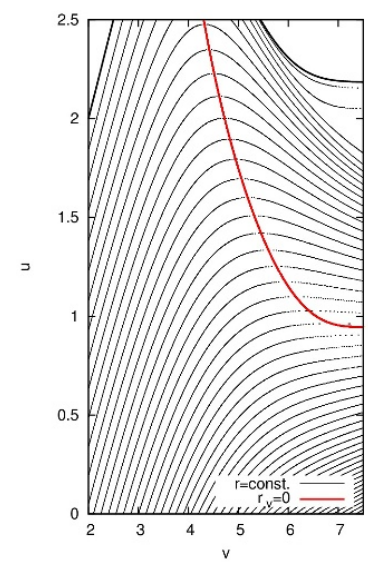

(a)

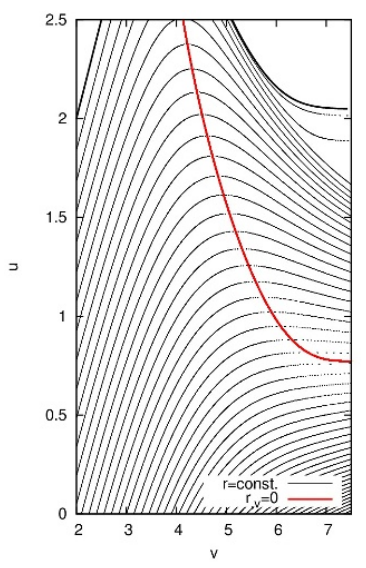

(b)

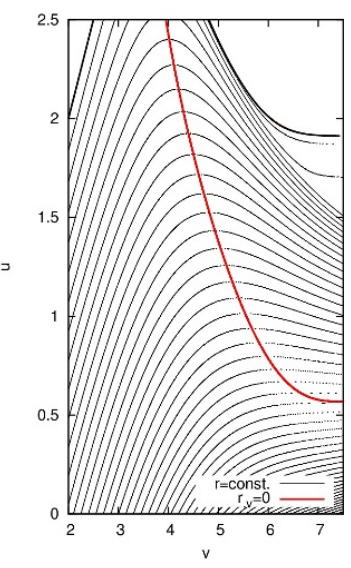

(c)

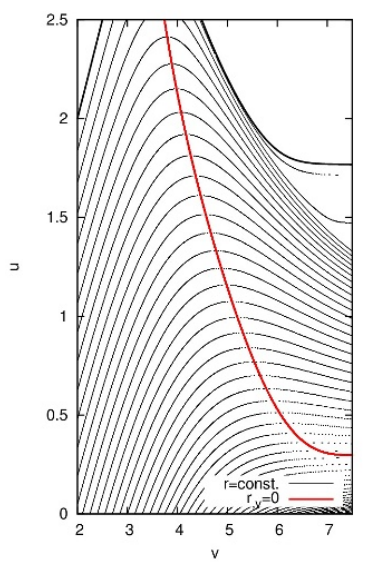

(d)

Figure 11. (color online) Penrose diagrams of spacetimes formed during the collapse of the electrically charged scalar field of the amplitude $\tilde{p}_{s}=0.6$ in the presence of dark matter with $m^{2}=0.1$ and $\tilde{p}_{h}$ equal to (a) $10^{-3}$, (b) 0.1 , (c) 0.15 and (d) 0.2 .

and $\lambda_{D M}$ do not play a significant role during the process. The latter result is an extension of the previous attempt to include the $\chi^{4}$-type interactions in the gravitational collapse mentioned in [129], which was made for the evolution of a real scalar field without electric charge. The results of the conducted simulations will be presented for $\alpha_{D M}=10^{-3}$ and $\lambda_{D M}=0.1$. The course of the $S F-D M$ evolution was also interpreted regarding the strength of a gravitational self-interaction of the evolving fields by changing the values of their initial amplitudes $\tilde{p}_{h}$ and $\tilde{p}_{s}$.

\subsection{Spacetime structures}

The Penrose diagrams of spacetimes formed during the $S F-D M$ collapse with a varying amplitude $\tilde{p}_{h}$ are presented in figures 11 and 12 for $m^{2}=0.1$ and the electrically charged scalar field amplitude $\tilde{p}_{s}$ equal to 0.6 and 0.2 , respectively. The structures of spacetimes, which stem from the process in the absence of dark energy are shown in figure 2 . The former is the dynamical Reissner-Nordström spacetime and the latter is non-singular.

In the case of $\tilde{p}_{s}=0.6$, all the resulting spacetimes are dynamical Reissner-Nordström spacetimes for all initial amplitudes of the complex field, which constitutes the dark matter model. The central spacelike singularity at $r=0$ is surrounded within each of them by a single apparent horizon situated along the $r_{, v}=0$ line, which settles down along an event horizon as $v \rightarrow \infty$. The Cauchy horizon is located at future null infinity $v=\infty$. An increasing value of $\tilde{p}_{h}$ results in an earlier formation of a black hole in terms of retarded time. When $\tilde{p}_{s}$ equals 0.2 , the obtained spacetime is non-singular for values of $\tilde{p}_{h}$ not exceeding 0.135 or contains a dynamical Reissner-Nordström black hole for its larger values. This result is particularly interesting due to the role played by dark matter in this case. Although the self-interaction of an electrically charged scalar field is too weak to form a black hole (figure 2a), the presence of dark matter enables the emergence of a singular spacetime. The appearance of the singularity is clearly related to the dark matter ac- 


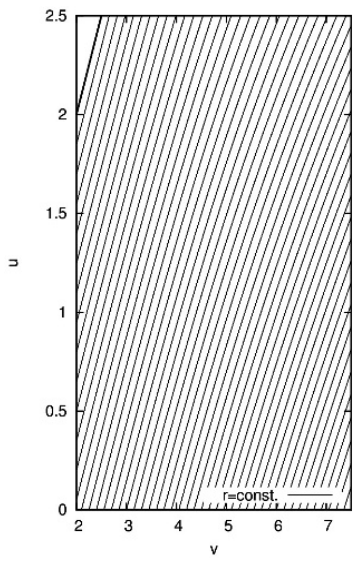

(a)

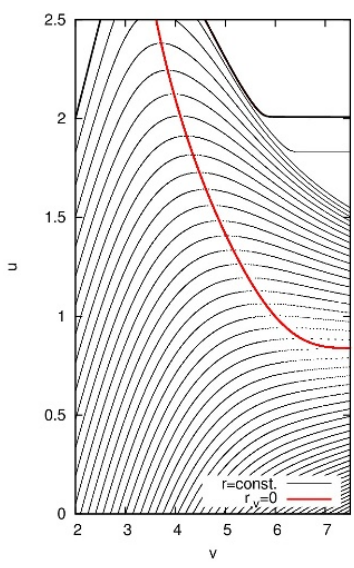

(b)

Figure 12. (color online) Penrose diagrams of spacetimes formed during the $S F-D M$ evolution with $\tilde{p}_{s}=0.2, m^{2}=0.1$ and $\tilde{p}_{h}$ equal to (a) 0.1 and (b) 0.4 .

tion during the collapse, but the existence of a Cauchy horizon is strictly connected with the charge associated with the scalar field coupled with the Maxwell field.

The spacetimes formed during the discussed collapse for $\tilde{p}_{s}=0.6, \tilde{p}_{h}=0.1$ and several negative values of $m^{2}$ are shown in figure 13. For $m^{2}$ larger than -3.85 the dynamical Reissner-Nordström spacetimes form, while for its smaller values the Cauchy horizon is absent in the spacetime and hence dynamical Schwarzschild black holes stem from the evolution. In the latter case, the central $r=0$ spacelike singularity is surrounded by an apparent horizon $r_{, v}=0$, which coincides with an event horizon at $v \rightarrow \infty$. The Cauchy horizon does not exist, because the $r=$ const. lines do not settle along $u=$ const. hypersurfaces as $v \rightarrow \infty$. For $m^{2}>0$ all the spacetimes are of the ReissnerNordström type. The non-zero vev of the dark matter complex scalar field, i.e., the existence of dark matter in the form of a massive gauge boson (called $Z^{\prime}$ or dark photon) during the course of the studied process for $m^{2}<-3.85$, results in the disappearance of a Cauchy horizon and favors the formation of a simpler Schwarzschild-type spacetime.

\subsection{Fields behavior in spacetimes}

The components of the stress-energy tensor (2.24) calculated for the spacetimes formed during the $S F-D M$ collapse characterized by the amplitudes $\tilde{p}_{s}=0.6, \tilde{p}_{h}=0.1$ and $m^{2}$ equal to -1 and -4 are presented in figure 14 (the respective spacetime structures were presented in figures 13a and 13d). The $(v u)$-distributions of the moduli of the electrically charged scalar field and the dark matter complex scalar field for the same evolutions are shown in figures 15 and 16.

Both $T_{u u}$ and $T_{v v}$ components of the stress-energy tensors are positive nearby the singular $r=0$ lines in the presented spacetimes. Hence, the null energy condition (4.1) is fulfilled in the vicinity of the central singularities. They do not bifurcate into wormhole throats and black holes form during the $S F-D M$ evolution. Similar conclusions stem 


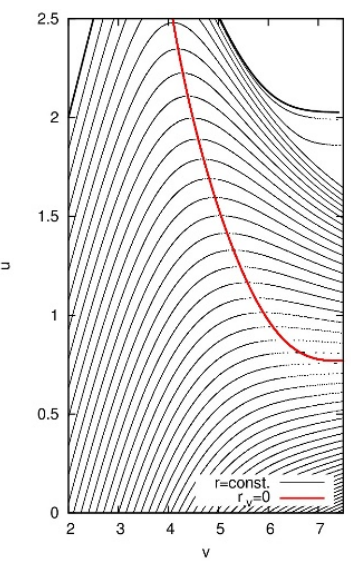

(a)

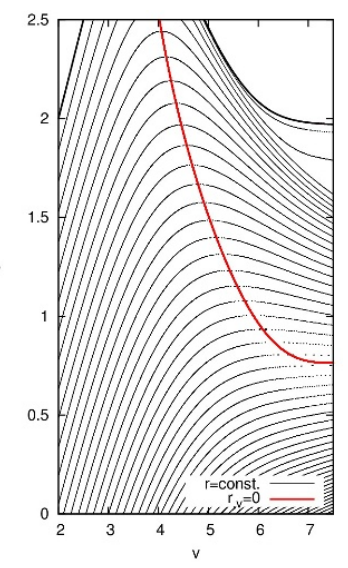

(b)

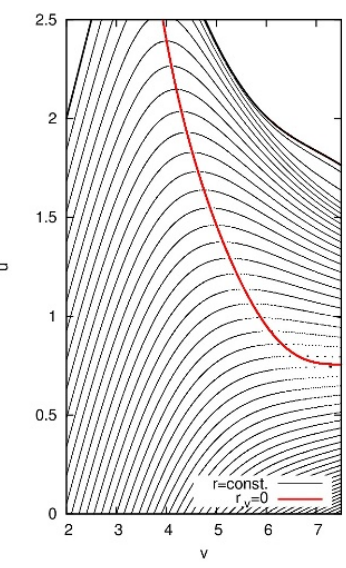

(c)

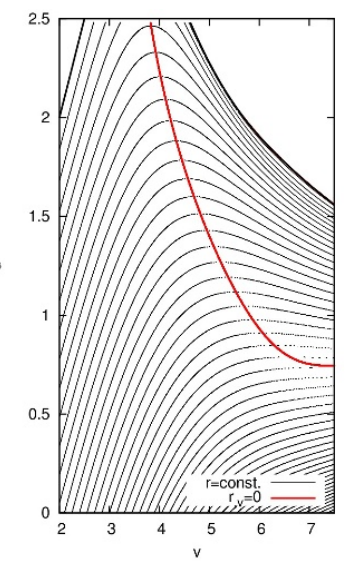

(d)

Figure 13. (color online) Penrose diagrams of spacetimes formed during the $S F-D M$ collapse with $\tilde{p}_{s}=0.6, \tilde{p}_{h}=0.1$ and $m^{2}$ equal to (a) -1 , (b) -2 , (c) -3 and (d) -4 .

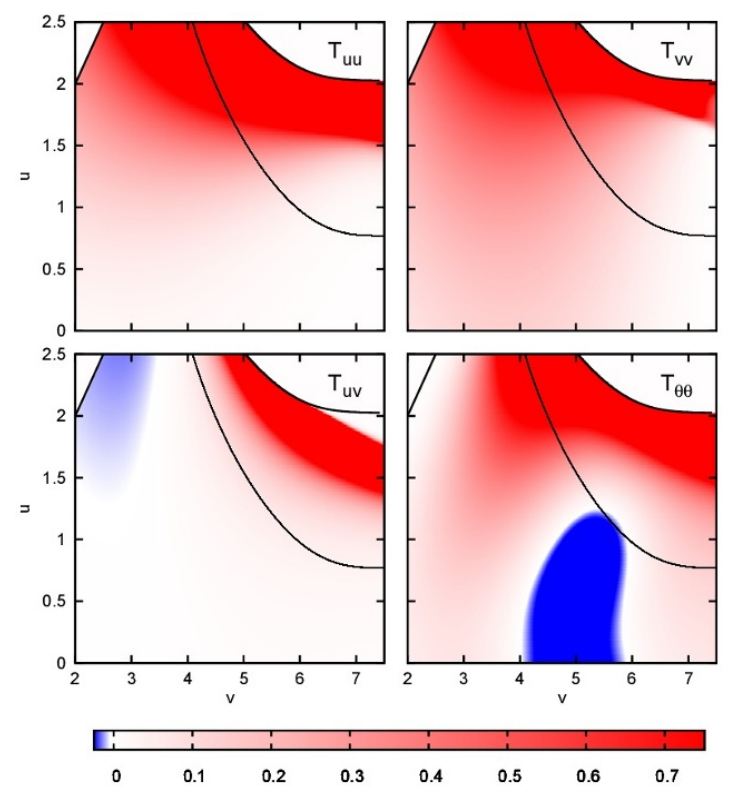

(a)

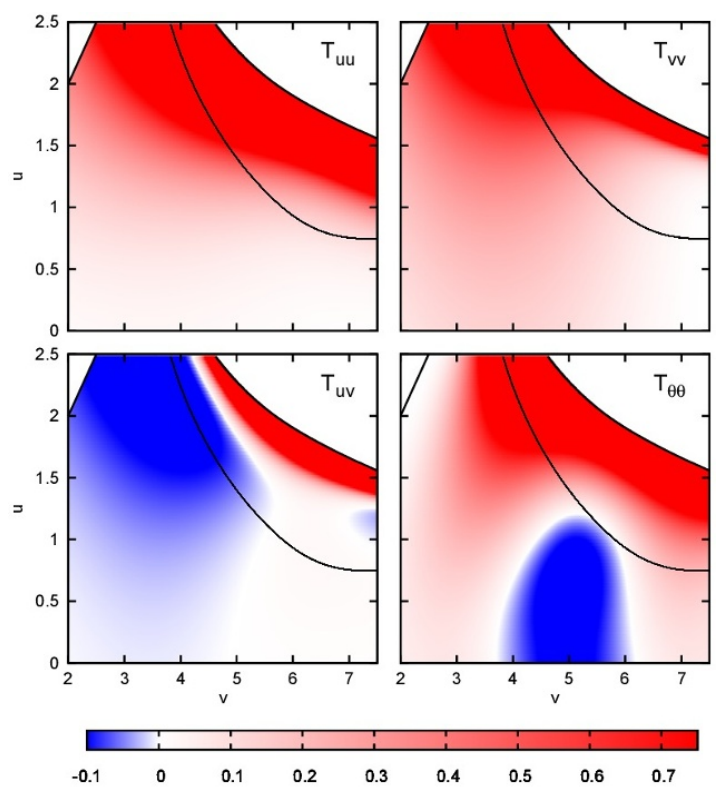

(b)

Figure 14. (color online) The stress-energy tensor components $(2.25)-(2.28)$ in the $(v u)$-plane for $S F-D M$ evolutions with $\tilde{p}_{s}=0.6, \tilde{p}_{h}=0.1$ and $m^{2}$ equal to (a) -1 and (b) -4 .

from the analysis of the stress-energy tensor components for singular spacetimes presented in figures 11 and 12 .

An inspection of the moduli of the field functions reveals that the type of the forming spacetime depends on their values in the vicinity of $r=0$ as $v \rightarrow \infty$. A significant value of the modulus of an electrically charged scalar field in this region, three orders of magnitude bigger than in the remaining spacetime, accompanied by a small value of the other field 


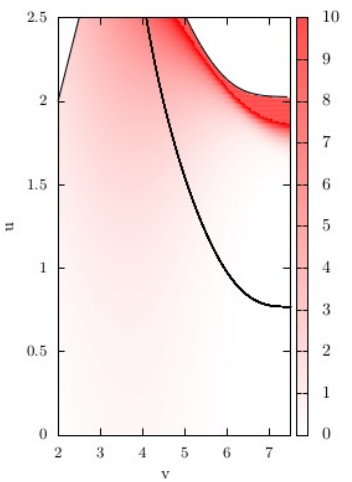

(a)

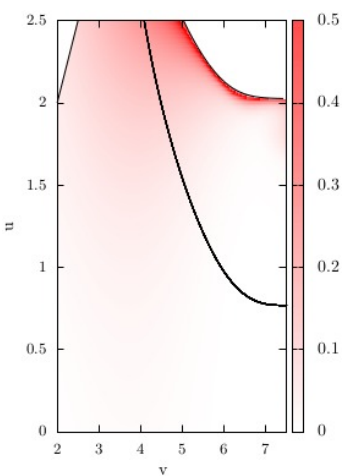

(b)

Figure 15. (color online) The $(v u)$-distribution of the moduli of (a) the electrically charged scalar field, $|\psi|$, and (b) the dark matter complex scalar field, $|\chi|$, for the $S F-D M$ evolution with $\tilde{p}_{s}=0.6$, $\tilde{p}_{h}=0.1$ and $m^{2}=-1$.

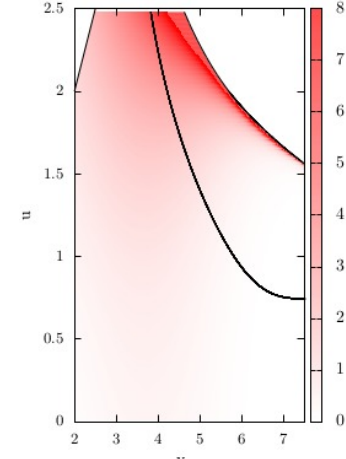

(a)

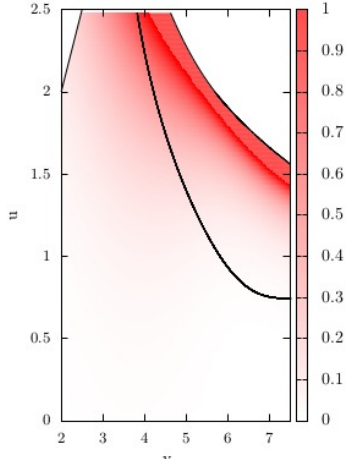

(b)

Figure 16. (color online) The (vu)-distribution of (a) $|\psi|$ and (b) $|\chi|$ for the $S F-D M$ evolution with $\tilde{p}_{s}=0.6, \tilde{p}_{h}=0.1$ and $m^{2}=-4$.

modulus, results in the formation of a dynamical Reissner-Nordström spacetime. It is connected with the repulsive character of the scalar field due to the electric charge associated with it. On the other hand, when the modulus of the dark matter complex scalar field is bigger at $v \rightarrow \infty$ nearby $r=0$ (two orders of magnitude in relation to the rest of the spacetime in the considered case), the importance of the other field is reduced and the dynamical Schwarzschild spacetime emerges. Such behavior of dark matter during the gravitational collapse is consistent with its action on a cosmological scale, where it tends to concentrate in confined areas due to the attractive character of its gravitational interaction. It is worth noting, that in the regime of the selected dark matter model, the described behavior is related to the change in potential (the value of $m^{2}$ ), not in the gravitational self-interaction strength of the complex field (the amplitude $\tilde{p}_{h}$ ). 


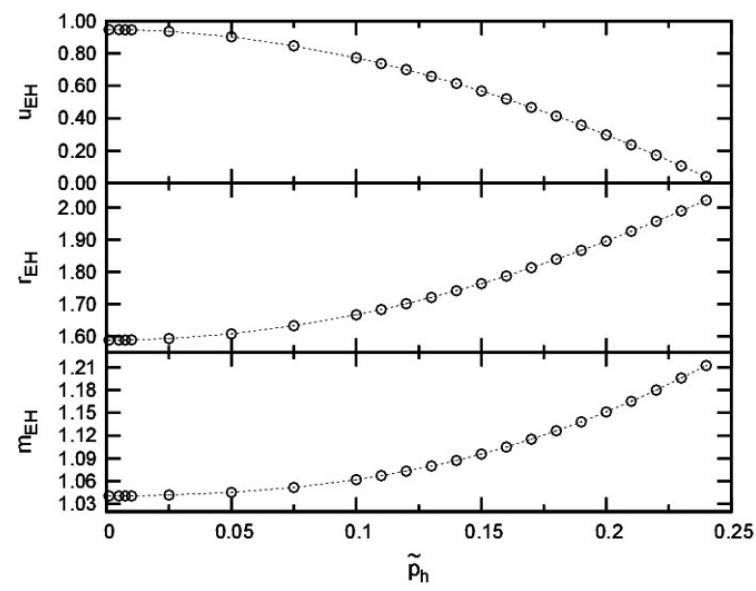

(a)

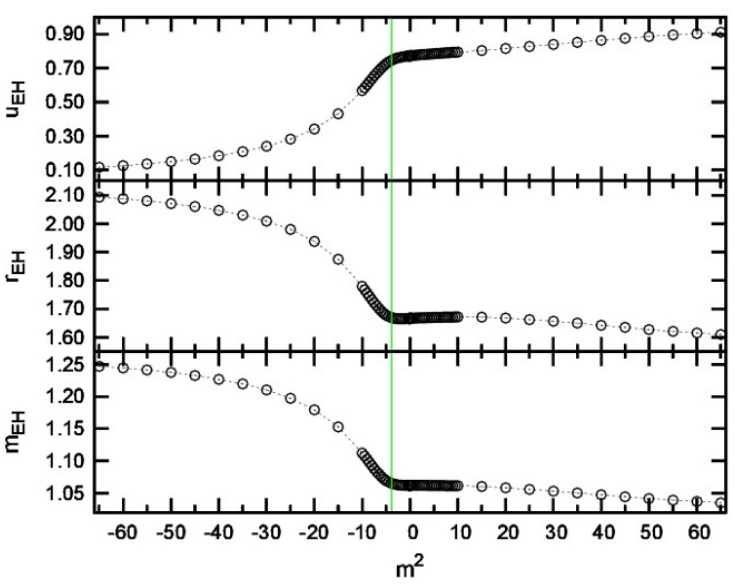

(b)

Figure 17. (color online) The $u$-locations of the event horizons, $u_{E H}$, the masses, $m_{E H}$, and the radii, $r_{E H}$, of black holes formed during the $S F-D M$ collapse as functions of (a) $\tilde{p}_{h}$ with $m^{2}=0.1$ and (b) $m^{2}$ with $\tilde{p}_{h}=0.1$. The green line indicates the border between spacetimes of the Schwarzschild $\left(m^{2}<-3.85\right)$ and Reissner-Nordström $\left(m^{2} \geqslant-3.85\right)$ types.

\subsection{Characteristics of the formed black holes}

The dependence of the event horizon $u$-locations, the masses and the radii of black holes formed during the collapse of an electrically charged scalar field accompanied by dark matter on $\tilde{p}_{h}$ and $m^{2}$ is depicted in figure 17. The maximal value of the initial amplitude of the dark matter scalar field was selected so that for all investigated cases the collapse begins outside of the event horizon.

The black holes form earlier in terms of retarded time and their masses and radii increase as $\tilde{p}_{h}$ gets bigger (figure 17a). When $\mathrm{m}^{2}$ increases the moment of the event horizon formation appears later in terms of $u$ and the black hole masses and radii decrease with an inflection nearby $m^{2}=-3.85$, which is the point of the change between the ReissnerNordström and Schwarzschild spacetime structures (figure 17b).

\section{Collective effect of dark components on the considered collapse}

Similarly to the evolutions described in section 5, the collapse of an electrically charged scalar field with both dark energy and dark matter will be presented for the values of $\alpha_{D M}$ and $\lambda_{D M}$ equal to $10^{-3}$ and 0.1 , respectively. The altering of the initial amplitudes $\tilde{p}_{s}$, $\tilde{p}_{h}$ and $\tilde{p}_{k}$ is equivalent to changing the strength of the particular field gravitational selfinteraction within the examined physical system.

\subsection{Spacetime structures}

The structures of spacetimes formed in the course of the $S F-D E-D M$ collapse for $\tilde{p}_{s}=0.6$, $\tilde{p}_{k}=0.15, m^{2}=0.1$ and the varying amplitude of the dark matter complex scalar field are 


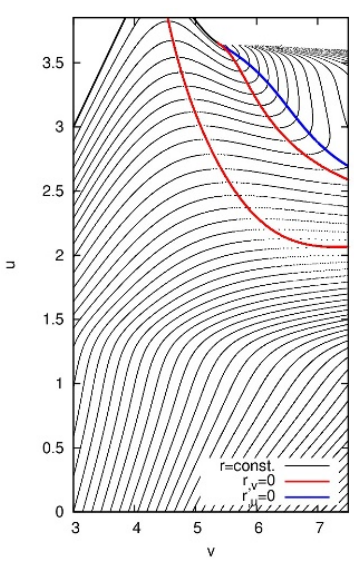

(a)

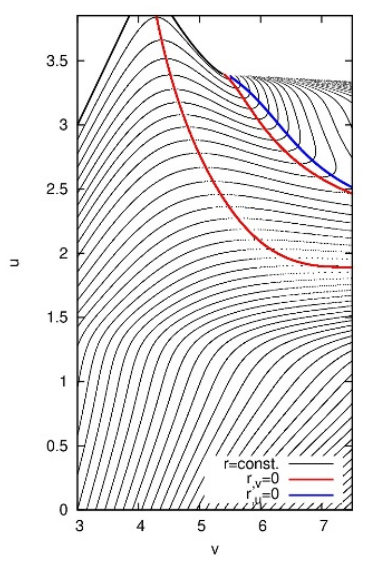

(b)

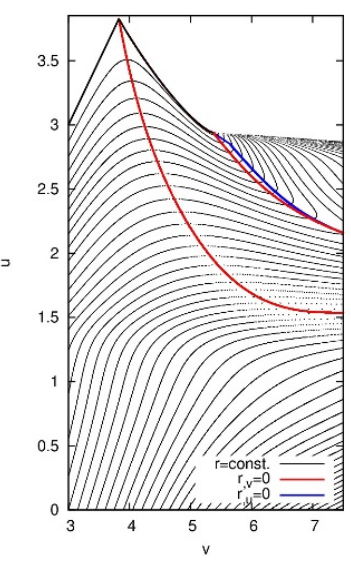

(c)

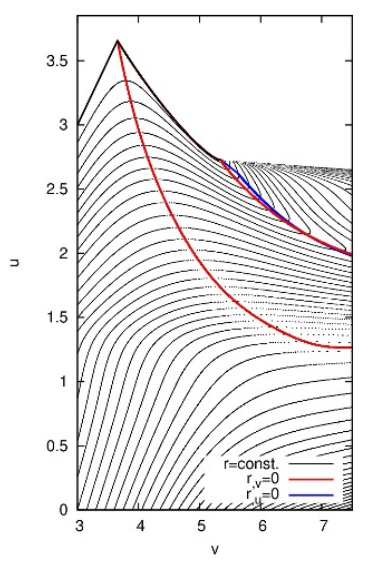

(d)

Figure 18. (color online) Penrose diagrams of spacetimes formed during the gravitational evolution of the electrically charged scalar field of the amplitude $\tilde{p}_{s}=0.6$ in the presence of the phantom scalar field with $\tilde{p}_{k}=0.15$ and dark matter, whose model is described by the parameters $m^{2}=0.1$ and $\tilde{p}_{h}$ equal to (a) $10^{-3}$, (b) 0.1 , (c) 0.2 and (d) 0.25 .

presented in figure 18. The obtained spacetimes contain dynamical wormholes. The central spacelike $r=0$ singularity bifurcates into two wormhole throats located along the lines $r_{, v}=0$ and $r_{, u}=0$ as $v \rightarrow \infty$. The whole is surrounded by an apparent horizon $r_{, v}=0$, which coincides with the event horizon in the region, where the spacetime settles down after the dynamical stage of the process. The value of $\tilde{p}_{h}$ does not influence the type of the emerging spacetime, but it causes the event horizon to form earlier in terms of retarded time.

The Penrose diagrams of spacetimes which stem from the studied collapse with a varying phantom field amplitude with $\tilde{p}_{s}=0.6, \tilde{p}_{h}=0.15$ and $m^{2}=0.1$ are shown in figure 19. The spacetimes contain either wormholes or naked singularities. The former emerge for $\tilde{p}_{k}$ smaller than 0.23 and the latter for its larger values. The $u$-location of the event horizon increases with the value of the altering amplitude. The gravitational self-interaction strength of the phantom field decides about the type of a spacetime structure, which forms in the process.

Figure 20 presents the results of the considered evolution for different negative values of the parameter $m^{2}$, when the initial field amplitudes are $\tilde{p}_{s}=0.6, \tilde{p}_{k}=0.15$ and $\tilde{p}_{h}=0.15$. There exist dynamical wormholes in the emerging spacetimes for $m^{2} \geqslant-2.1$ and Schwarzschild black holes for its smaller values. The collapse with positive $m^{2}$ always results in a spacetime, which contains a dynamical wormhole.

The above results indicate that when the field gravitational self-interaction strength is concerned, the role of dark energy coexists with the influence of dark matter. The dark energy component decides what type of an object forms. Dark matter does not interfere with the structure type, but regulates the amount of time which is needed for the intrinsic object to form. When the impact of the dark matter potential is considered, it turns out that the value of $\mathrm{m}^{2}$ influences the observed structure by controlling whether the wormhole throats remain open in the spacetime. Their absence results in the formation 


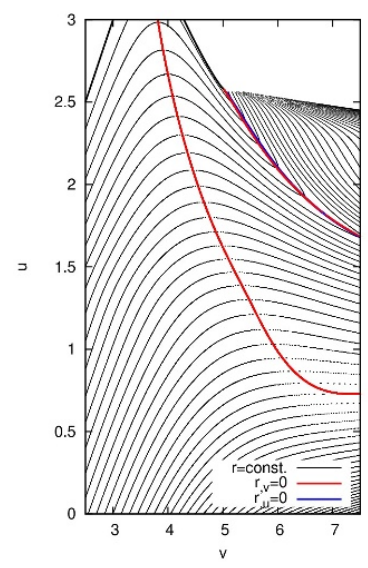

(a)

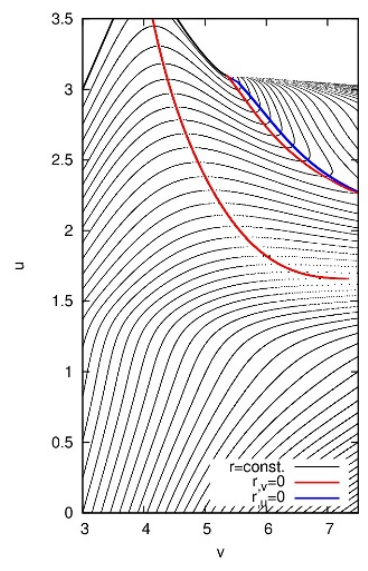

(b)

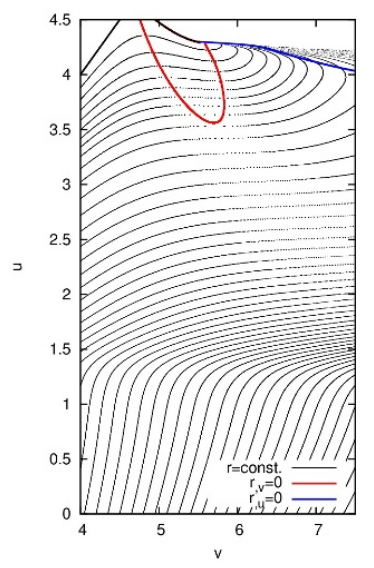

(c)

Figure 19. (color online) Penrose diagrams of spacetimes formed during the collapse of the electrically charged scalar field of the amplitude $\tilde{p}_{s}=0.6$ in the presence of dark matter with $\tilde{p}_{h}=0.15$, $m^{2}=0.1$ and the phantom scalar field with $\tilde{p}_{k}$ equal to (a) 0.05 , (b) 0.14 and (c) 0.25 .

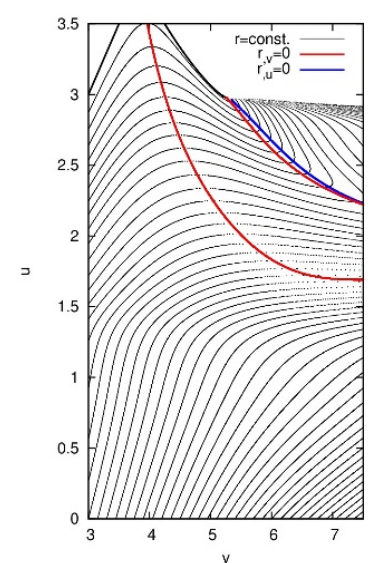

(a)

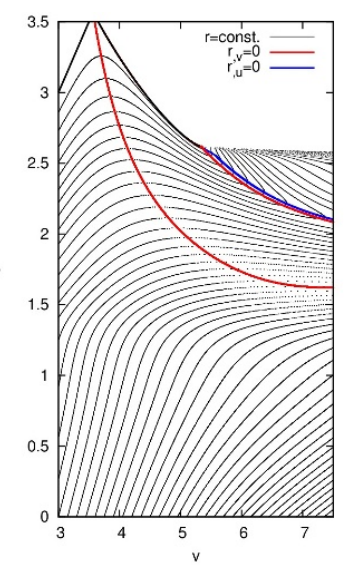

(b)

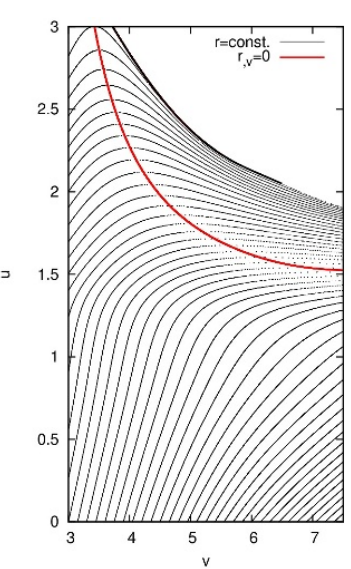

(c)

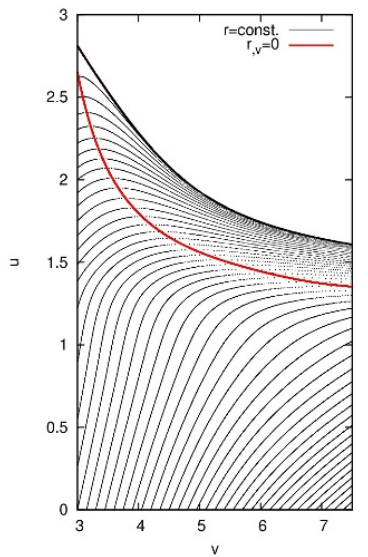

(d)

Figure 20. (color online) Penrose diagrams of spacetimes formed during the $S F-D E-D M$ collapse with $\tilde{p}_{s}=0.6, \tilde{p}_{k}=0.15, \tilde{p}_{h}=0.15$ and $m^{2}$ equal to (a) -1 , (b) -2 , (c) -3 and (d) -5 .

of a Schwarzschild spacetime and the existence of a central singularity even after the dynamical phase of the collapse is finished. It should be emphasized that the dark sector suppresses the natural tendency of an electrically charged scalar field to form a dynamical Reissner-Nordström spacetime in the course of the gravitational collapse.

\subsection{Fields behavior in spacetimes}

The $(v u)$-distributions of the stress-energy tensor components (2.25)-(2.28) for the dynamical spacetimes emerging from the $S F-D E-D M$ evolutions characterized by the amplitudes $\tilde{p}_{s}=0.6, \tilde{p}_{k}=0.15, \tilde{p}_{h}=0.15$ and $m^{2}$ equal to -1 and -3 are presented in figure 21 (the respective spacetime structures were presented in figures 20a and 20c). The spacetime 


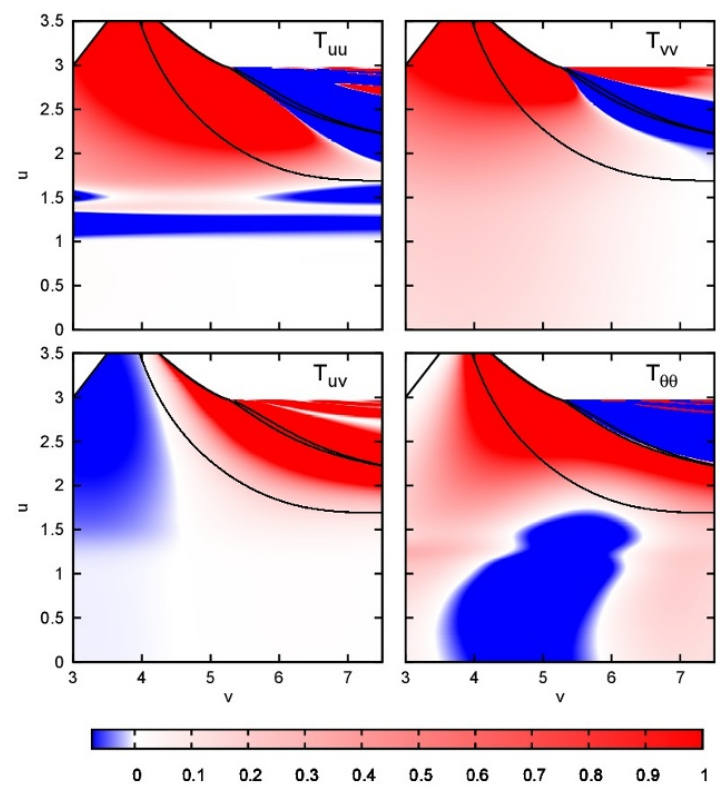

(a)

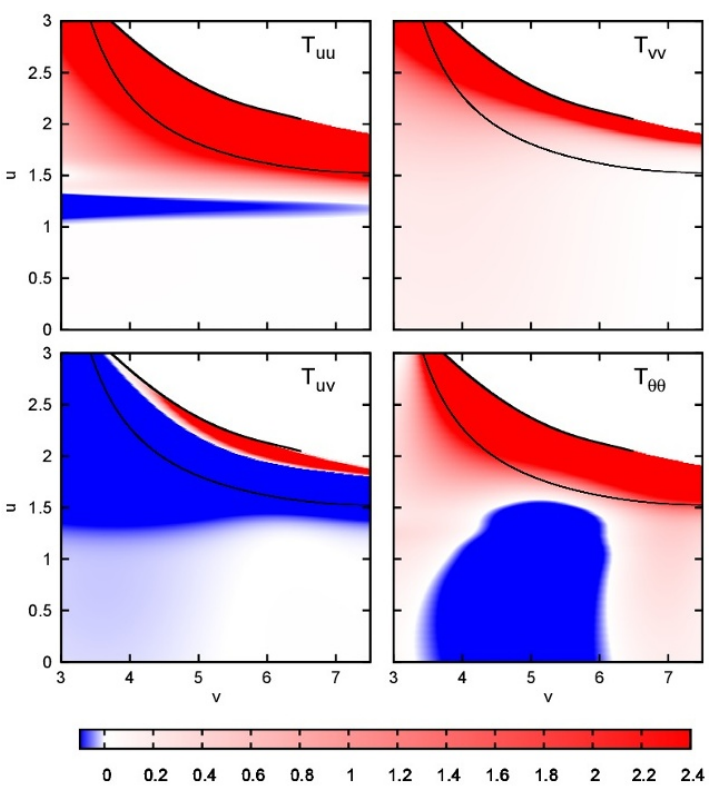

(b)

Figure 21. (color online) The stress-energy tensor components (2.25)-(2.28) in the $(v u)$-plane for $S F-D E-D M$ evolutions with $\tilde{p}_{s}=0.6, \tilde{p}_{k}=0.15, \tilde{p}_{h}=0.15$ and $m^{2}$ equal to (a) -1 and (b) -3 .

distributions of the moduli of the electrically charged scalar field, the dark matter complex scalar field and the phantom scalar field for the same cases are shown in figures 22 and 23.

The $T_{u u}$ and $T_{v v}$ components of the stress-energy tensors are negative in the vicinity of wormhole throats when $m^{2}=-1$. The null energy condition (4.1) is violated there. In the case of $m^{2}=-3$ the components are positive nearby $r=0$ and hence a black hole, not a dynamical wormhole arises in the spacetime. The obtained result strengthens the conclusion presented in section 4.2 that the violation of NEC is needed for the formation of wormholes during dynamical evolutions. Clearly, this statement is valid even for complicated matter-geometry systems evolutions, in which several types of interacting matter are involved.

Similarly to the $S F-D M$ case, the obtained spacetime type depends on the values of the evolving field functions in the vicinity of $r=0$. The formation of a dynamical wormhole stems from a significant value of the modulus of the phantom scalar field in this region as $v \rightarrow \infty$ (four orders of magnitude bigger than in the remaining spacetime) accompanied by a small value of the dark matter field modulus. When the modulus of the dark matter complex scalar field is bigger at $v \rightarrow \infty$ nearby $r=0$ (three orders of magnitude in relation to the rest of the spacetime in the considered case), the importance of the remaining fields is reduced and the dynamical Schwarzschild spacetime emerges. The structure type and the fields behavior in a spacetime depend on the value of the parameter $m^{2}$, not the initial amplitude of either of the collapsing fields. 


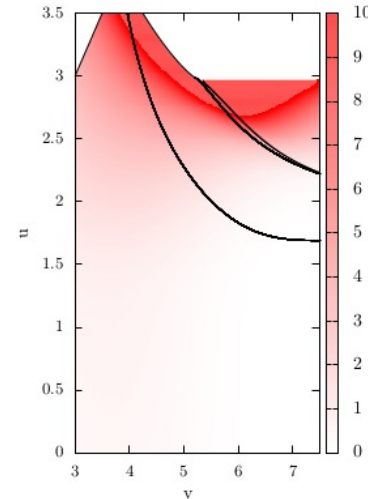

(a)

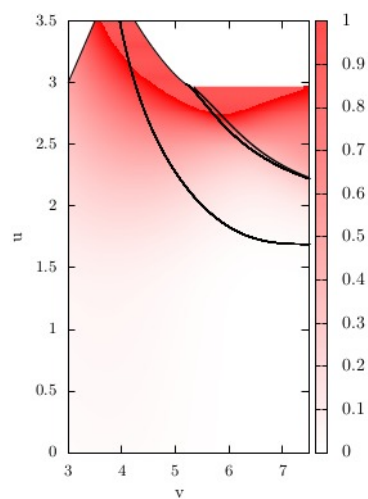

(b)

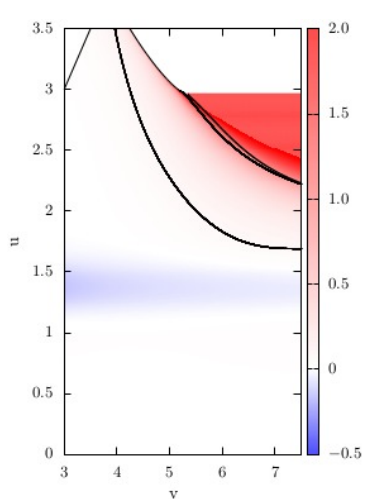

(c)

Figure 22. (color online) The (vu)-distribution of (a) the moduli of the electrically charged scalar field, $|\psi|$, (b) the moduli of the dark matter complex scalar field, $|\chi|$, and (c) the phantom scalar field, $\phi$, for the $S F-D E-D M$ evolution with $\tilde{p}_{s}=0.6, \tilde{p}_{k}=0.15, \tilde{p}_{h}=0.15$ and $m^{2}=-1$.

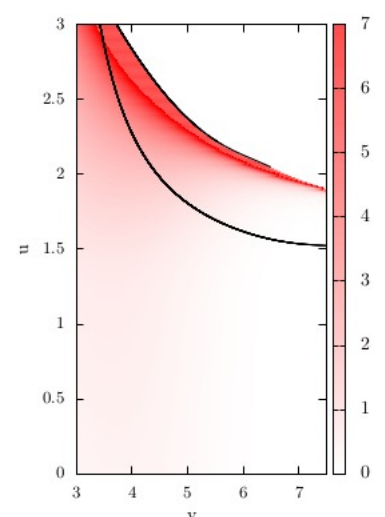

(a)

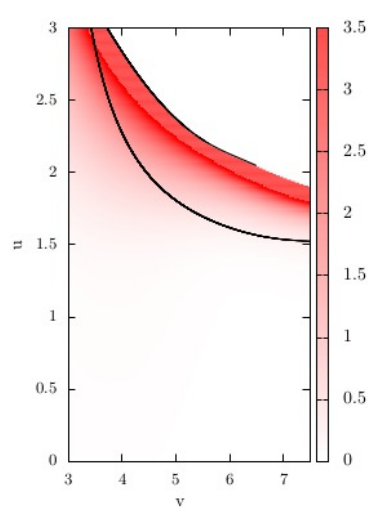

(b)

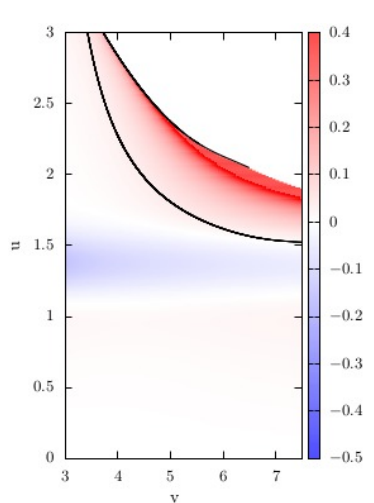

(c)

Figure 23. (color online) The (vu)-distribution of (a) $|\psi|$, (b) $|\chi|$ and (c) $\phi$ for the SF-DE-DM evolution with $\tilde{p}_{s}=0.6, \tilde{p}_{k}=0.15, \tilde{p}_{h}=0.15$ and $m^{2}=-3$.

\subsection{Properties of the emerging objects}

The influence of $\tilde{p}_{h}, \tilde{p}_{k}$ and $m^{2}$ on the event horizon $u$-locations, the masses and the radii of objects formed during the gravitational evolution of an electrically charged scalar field accompanied by dark matter and dark energy is shown in figure 24. The maximal value of the amplitude $\tilde{p}_{h}$ was determined by the event horizon location in the spacetime so as to begin the collapse outside it. The maximal considered value of the phantom scalar field amplitude was the biggest value not leading to the formation of a naked singularity in spacetime.

As the dark matter complex scalar field amplitude increases, the wormholes appear earlier in $u$, their radii increase and masses decrease up to $\tilde{p}_{h}=0.23$ and increase for larger $\tilde{p}_{h}$ (figure 24a). In the case of an increasing $\tilde{p}_{k}$, the values describing all the properties increase, apart from $r_{E H}$ for values of the amplitude exceeding 0.21 (figure 24b). Just as in the case 


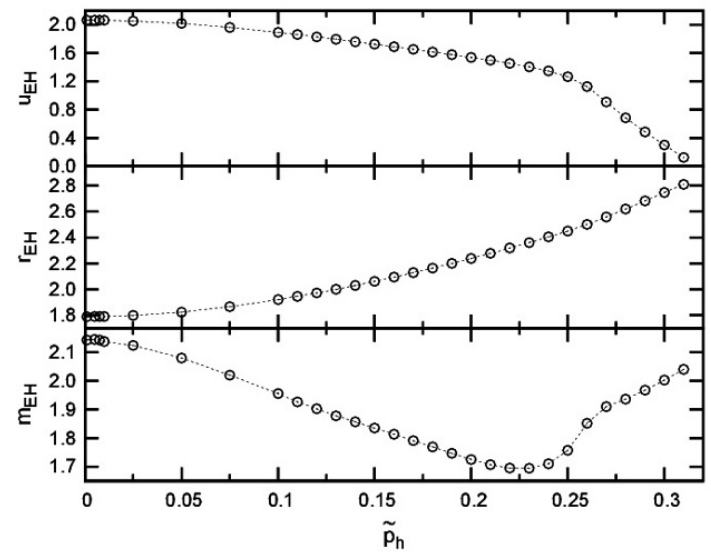

(a)

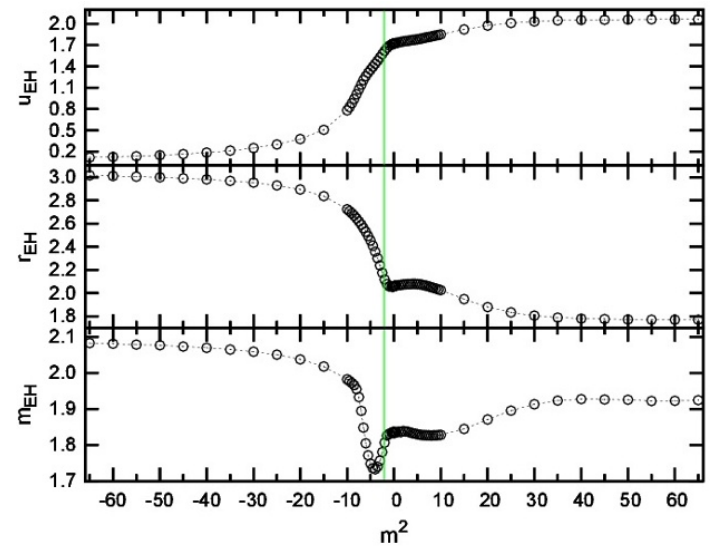

(c)

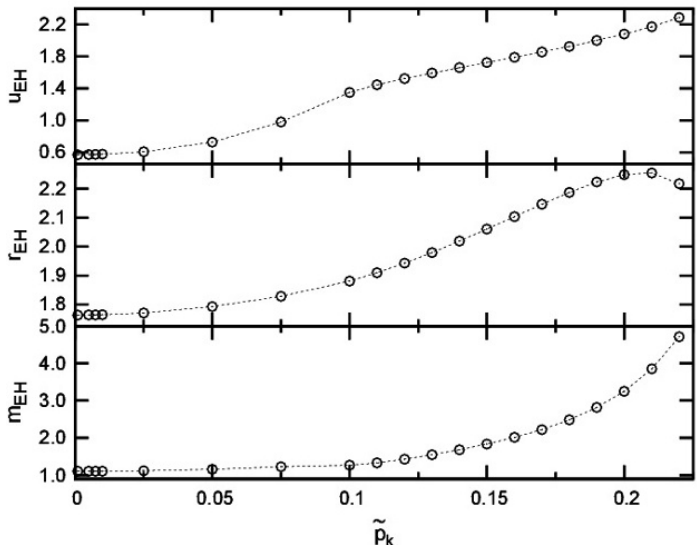

(b)

Figure 24. (color online) The $u$-locations of the event horizons, $u_{E H}$, the masses, $m_{E H}$, and the radii, $r_{E H}$, of the objects formed during the $S F-D E-D M$ collapse as functions of (a) $\tilde{p}_{h}$ with $\tilde{p}_{k}=0.15, m^{2}=0.1$, (b) $\tilde{p}_{k}$ with $\tilde{p}_{h}=0.15, m^{2}=0.1$ and (c) $m^{2}$ with $\tilde{p}_{k}=0.15, \tilde{p}_{h}=0.15$. The green line indicates the border between spacetimes containing black holes of the Schwarzschild type $\left(m^{2}<-2.1\right)$ and wormholes $\left(m^{2} \geqslant-2.1\right)$.

of the $S F-D E$ system, such behavior indicates the upcoming formation of a naked singularity in the spacetime. When $m^{2}$ increases the event horizon forms later in terms of retarded time and the wormhole radii decrease with an inflection nearby $m^{2}=-2.1$, which is the point of the change between the wormhole and Schwarzschild spacetime structures (figure 24c). The behavior of masses is more complicated, as apart from the inflection around $m^{2}=-2.1$, there exists a minimal mass for $m^{2}=-3.9$. The non-monotonic behavior of the described characteristics is related to the fact that the investigated system consists of multiple components and the observed phenomena are a compromise among the tendencies characteristic for particular evolving matter types. 


\section{Conclusions}

The influence of dark matter and dark energy on the electrically charged scalar field collapse was investigated. Dark matter was described by a complex scalar field with a quartic potential, coupled to a U(1)-gauge field. Dark energy was modeled by a scalar field coupled to gravity in a phantom manner. The exponential relation of the scalar field with dark energy originated from the low-energy limit of the string theory. Dark matter was coupled with the electrically charged scalar field through a kinetic coupling between the two gauge fields present in the system.

The existence of phantom matter in spacetime can result in the formation of naked singularities or dynamical wormholes during the gravitational collapse of an electrically charged scalar field. The latter objects have two throats related to two travel directions and are surrounded by event horizons. The values of the phantom field are considerably bigger in the spacetime region situated nearby wormhole throats, in comparison to the surrounding area. This accumulation leads to the violation of the null energy condition, which is essential for the dynamical wormhole formation and the stabilization of its throats. The masses of wormholes, the radii of event horizons and wormhole throats increase when the amplitudes of both phantom and electrically charged scalar fields increase. The event horizon, wormhole throats and singularity origin appear later in terms of retarded time as $\tilde{p}_{k}$ increases while $\tilde{p}_{s}$ is constant and at smaller values of the $u$-coordinate in the opposite case.

In the obtained wormhole spacetimes spacelike singularities situated along $r=0$ exist during the dynamical stage of the evolution. They turn into wormhole throats as the spacetime tends towards the final stage of the collapse. This suggests that the considered spacetime shortcuts ensue from the process, during which a black hole gains exotic matter of negative energy. Thus the existence of duality between black holes and wormholes was confirmed in the course of fully non-linear computations.

The spacetimes resulting from the examined collapse in the presence of dark matter are either non-singular or contain black holes, depending on the gravitational self-interaction strengths of both evolving fields, that is their initial amplitudes $\tilde{p}_{s}$ and $\tilde{p}_{h}$. The type of an emerging object depends on the value of the square of mass parameter from the dark matter model. In general, the non-zero vev of the dark matter complex scalar field favors the formation of dynamical Schwarzschild spacetimes for $m^{2}<-3.85$, while for the vanishing vev the dynamical Reissner-Nordström black holes appear.

The null energy condition is fulfilled in the vicinity of singularities in the emerging spacetimes, hence the stabilization of wormhole throats is impossible and such objects do not form. The values of the moduli of the electrically charged scalar field and the dark matter complex scalar field are considerably bigger nearby $r=0$, where $v \rightarrow \infty$, than in the remaining regions in spacetimes of Reissner-Nordström and Schwarzschild types, respectively. This indicates a repulsive character of the former and an attractive character of the latter field.

The moment of a black hole formation, i.e., an appearance of an event horizon in terms of retarded time, as well as the black hole masses and radii are controlled by the values of $\tilde{p}_{h}$ and $m^{2}$. The event horizon $u$-locations increase and the remaining characteristics 


\begin{tabular}{|c|c|c|c|}
\hline \multirow{2}{*}{ Evolution } & $\tilde{p}_{k} \nearrow$ & $\tilde{p}_{h} \nearrow$ & $m^{2} \nearrow$ \\
\hline \multirow{2}{*}{$S F-D E$} & $W H \rightarrow N S \rightarrow n s[16]$ & & - \\
& $u_{E H} \nearrow$ & - & \\
\hline \multirow{3}{*}{$S F-D M$} & $r_{E H} \nearrow m_{E H} \nearrow$ & & $R N \rightarrow S$ \\
& - & $u_{E H} \searrow$ & $u_{E H} \nearrow$ \\
& & $r_{E H} \nearrow m_{E H} \nearrow$ & $r_{E H} \searrow m_{E H} \searrow$ \\
\multirow{2}{*}{$S F-D E-D M$} & $W H \rightarrow N S$ & $W H$ & $W H \rightarrow S$ \\
& $u_{E H} \nearrow$ & $u_{E H} \searrow$ & $u_{E H} \nearrow$ \\
& $r_{E H} \searrow m_{E H} \nearrow$ & $r_{E H} \nearrow m_{E H} \searrow \nearrow$ & $r_{E H} \searrow m_{E H} \searrow \nearrow$ \\
\hline
\end{tabular}

Table 1. Spacetime structures formed during the gravitational collapse of an electrically charged scalar field with $\tilde{p}_{s}=0.6$ accompanied by dark energy and dark matter and the selected characteristics of the emerging objects. Symbols: $n s$ - non-singular spacetime, $S$ - Schwarzschild-type spacetime, $R N$ - Reissner-Nordström-type spacetime, $W H$ - wormhole, $N S$ - naked singularity, $u_{E H}-u$-location of an event horizon, $r_{E H}$ and $m_{E H}$ - radius and mass of the intrinsic object, respectively. Arrows: $\nearrow \searrow$ - an increase/decrease of a value of the particular characteristic or amplitude, $\rightarrow$ - the change of the spacetime structure while the particular parameter varies.

decrease as the dark matter complex scalar field initial amplitude raises. The opposite tendency is observed in the case of an increasing parameter $\mathrm{m}^{2}$.

The dark sector suppresses the tendency of a self-interacting electrically charged scalar field to form a dynamical Reissner-Nordström black hole during the gravitational evolution. In the case of its overall influence on the investigated process, the strength of the dark energy gravitational self-interaction prevails over the dark matter gravitational self-interaction strength. The dark energy component decides on the type of an object, while dark matter regulates the amount of time which is needed for it to form. The parameter $m^{2}$ of the dark matter potential influences the observed structures by controlling whether the wormhole throats appear in the spacetime. Their absence results in the formation of a Schwarzschild-type black hole, for the non-zero vev of the dark matter complex scalar field and $m^{2}<-2.1$.

In the spacetimes resulting from the $S F-D E-D M$ collapse the null energy condition is violated in the vicinity of wormhole throats and fulfilled nearby the singularities, just as in the previous cases. The formation of a wormhole requires values of a phantom scalar field considerably bigger nearby the throats in comparison to the rest of a spacetime. Large values of the modulus of the dark matter complex scalar field result in the emergence of a Schwarzschild-type object.

The behavior of $u$-locations, masses and radii as functions of $\tilde{p}_{h}, \tilde{p}_{k}$ and $m^{2}$ are complicated due to the fact that the investigated system consists of multiple components, each of which displays a different tendency during the evolution. The electrically charged scalar field favors the Reissner-Nordström black hole formation, dark energy supports the emergence of wormholes or naked singularities and dark matter tends to form the Schwarzschild- 


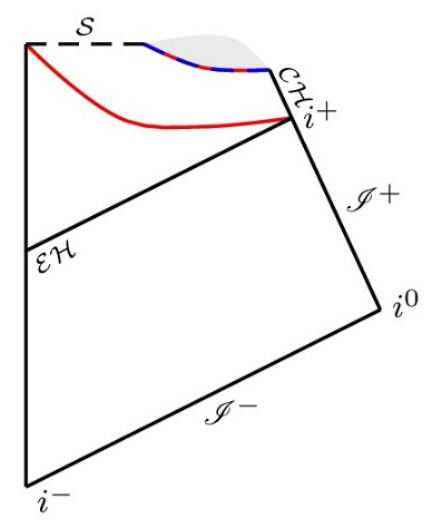

(a)

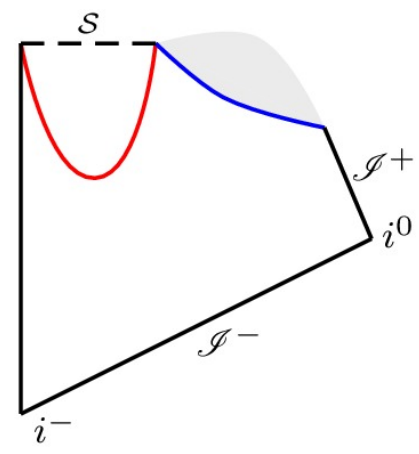

(b)

Figure 25. (color online) The Carter-Penrose diagrams of dynamical spacetimes, which contain (a) a wormhole and (b) a naked singularity. The red and blue solid lines are the $r_{, v}=0$ and $r_{, u}=0$ horizons, respectively. The red and blue dashed line symbolizes wormhole throats. The meaning of the symbols is the same as in figure 1 . The causal structure of the spacetime regions marked gray on the diagrams is impossible to interpret without any doubts due to the limitations of numerical computations in these areas.

type objects. The observed phenomena are a compromise among these tendencies characteristic for the involved matter types.

The changes introduced by the dark sector to the electrically charged scalar field gravitational collapse are presented in table 1. It summarizes the obtained results in the context of the role of particular parameters in the studied evolution for the initial amplitude of an electrically charged scalar field, which solely leads to the formation of a black hole. The obtained outcomes confirm the previous conclusions regarding the role of dark energy during the gravitational collapse. It does not prevent the formation of singular spacetimes during the dynamical evolution when it is accompanied by other types of matter, in particular dark matter $[18,20]$. The current studies also widened previous findings in this regard. They allowed us to describe the structures of objects existing in these singular spacetimes, which turned out to be wormholes, naked singularities or black holes of Schwarzschild or Reissner-Nordström types.

The Penrose diagrams of spacetimes, on which the spacetime structures were presented in the current paper, are related to the Carter-Penrose diagrams via conformal transformation, which preserves the causal structure of a spacetime. The Carter-Penrose diagrams of both static and dynamical Schwarzschild and Reissner-Nordström spacetimes can be found, e.g., in $[6,8,114]$. In figure 25 we present such diagrams for the dynamical wormhole and naked singularity spacetimes, which stem from the performed analyses of the dynamical formation of these objects.

Apart from investigations of spacetime structures formed during the collapse in the presence of the dark sector, other tantalizing questions are the mechanism of black hole formation and the behavior of matter accreting onto a nascent black hole taking the cosmological evolution into account. It was claimed that primordial black holes can 
accrete the surrounding dark energy, phantom energy or ghost condensate very effectively $[130,131]$. However, the conducted analyses neglected cosmic expansion.

Until now, there have been several attempts to find black hole solutions embedded in the expanding Universe, starting from the Einstein-Straus [132] and McVittie [133] solutions to the multi black hole Kastor-Traschen cosmological solution [134], which is a timedependent generalization of the Majumdar-Papapetrou one. An exact analytical black hole solution in the expanding Universe was derived within the Einstein-scalar-Maxwell system with two U(1)-gauge fields and an exponential potential of the scalar field [135]. The dilaton black hole on a thick brane and a cosmological brane black hole solutions were studied in $[136,137]$, while the time-dependent solution from compactification of intersecting branes in higher-dimensional unified theories was found in [138]. As was already stated in the Introduction, the dynamical solutions may behave quite differently in comparison to their stationary cousins. Some realistic attempts to analytically describe the accretion of a matter field onto an evolving black hole were conducted in [139], where a time-dependent scalar field and a time-dependent black hole were considered. In order to correctly account for the scalar field accretion, the expansion in terms of a slow roll parameter was implemented. The only initial assumptions about the geometry were its $\mathrm{SO}(3)$ symmetry and the time and radial dependence of the line element. Hence, the presented method did not require a direct assumption about the form of the underlying black hole metric.

The issues introduced above to be studied in the fully non-linear dynamical context are beyond of the scope of the presented research, as they require different methodology than the one employed in the paper. We hope to return to the announced problems in relation to the cosmological evolution of the Universe in the future researches.

\section{Acknowledgments}

A.N. was supported by the Polish National Science Centre under doctoral scholarship ETIUDA DEC-2013/08/T/ST2/00007 and partially by the Polish National Science Centre grant no. DEC-2014/15/B/ST2/00089. M.R. was partially supported by the Polish National Science Centre grant no. DEC-2014/15/B/ST2/00089. E.N. was supported by the Polish National Science Centre under postdoctoral scholarship FUGA DEC2014/12/S/ST2/00332.

\section{A Numerical computations}

The numerical simulations of the investigated process were conducted with the use of a modified version of the code, the specifics of which were presented in [14]. The scheme was broadened and adapted for the presently considered problem by including the phantom constant $\xi$ and the additional sector (2.52)-(2.55), which refers to dark matter. Due to the coupling between the $P_{\mu}$ and $A_{\mu}$ fields, the module covering the Maxwell field evolution (2.51) was also modified. The module governing the geometry dynamics $(2.44)-(2.47)$ was adjusted to the presently examined physical system. 
The inclusion of the additional sector in the code required posing adequate initial and boundary conditions for functions $z_{1}, z_{2}, h_{1}, h_{2}, w_{1}, w_{2}, T, \gamma, \kappa_{1}$ and $\kappa_{2}$. The first two evolved along $u$ according to equations $H_{(R e)}$ and $H_{(I m)}$. The dynamics of $h_{1}, h_{2}, w_{1}, w_{2}$, $T$ and $\gamma$ along $v$ was governed by equations $P 7, P 8, H_{(R e)}, H_{(I m)}, C 2$ and $C 1$, respectively. The values of the remaining two quantities were calculated on the basis of their definitions included in (2.35).

Initial conditions were posed on a null hypersurface denoted as $u=0$. The profiles of functions $h_{1}$ and $h_{2}$ were assigned according to (3.2). The values of $z_{1}$ and $z_{2}$ were calculated analytically using the relations $P 7$ and $P 8$. The behavior of the rest of the quantities, i.e., $w_{1}, w_{2}, T$ and $\gamma$, at the initial spacetime slice was resolved with the use of the threepoint Simpson method and the Newton method at the first point.

The line $u=v$ was the line of boundary conditions. Because of the fact that the collapsing physical system consists of a matter shell, the values of $T$ and $\gamma$ were equal to zero at the boundary, which refers to the non-singular $r=0$ line. The quantities $\kappa_{1}$ and $\kappa_{2}$ also vanished due to the equations $H_{(R e)}$ and $H_{(I m)}$. The flattening of the functions $h_{1}$ and $h_{2}$ was imposed on the boundary, i.e., $h_{1, r}$ and $h_{2, r}$ were equal to zero there. The values of the quantities $w_{1}$ and $w_{2}$ were the same as $z_{1}$ and $z_{2}$, respectively.

The accuracy of the numerical code was checked indirectly, due to the fact that no analytical solutions exist for the investigated process. The tests were performed for the evolutions initiated with parameters $\tilde{p}_{s}=0.6, \tilde{p}_{h}=0.15, m^{2}=0.1, \tilde{p}_{k}=0.14$ and $\tilde{p}_{s}=0.6$, $\tilde{p}_{h}=0.15, m^{2}=-3, \tilde{p}_{k}=0.15$. The respective spacetime structures are presented in figures $19 \mathrm{~b}$ and $20 \mathrm{c}$. For the purpose of testing the numerical code, these two cases will be referred to as evolutions 1 and 2, respectively. The analyses were done for spacetimes obtained within the $S F-D E-D M$ physical system, which was the most comprehensive among those discussed in the paper. Hence, the performed code accuracy checks covered the operation of all the constituent modules of the code.

The first test was based on checking the convergence of the obtained results. In order to monitor the convergence, the computations for evolutions 1 and 2 were conducted on four grids with integration steps being multiples of $10^{-4}$. A step of a particular grid was twice the size of a denser one. The convergence was examined on $u=$ const. hypersurfaces chosen for each case arbitrarily. The selected hypersurfaces were situated close to the emerging horizon, but in the region where the adaptive grid was yet inactive, which was necessary for performing a proper comparison of the outcomes.

The field functions along the selected hypersurfaces of constant $u$ are shown in figure 26. The areas in which the differences among functions obtained on various grids were most significant were magnified. The maximum observed discrepancy between the finest and coarsest grids was equal to $1.5 \%$. Figure 27 presents the linear convergence of the numerical code. The maximal divergence between the field profiles obtained on two grids with a quotient of integration steps equal to 2 and their respective doubles was $5.7 \%$. As expected, the errors became smaller linearly as the grid density increased. The overall convergence analysis revealed that both the algorithm and the numerical code were adequate for solving the system of equations (2.36)-(2.55). 


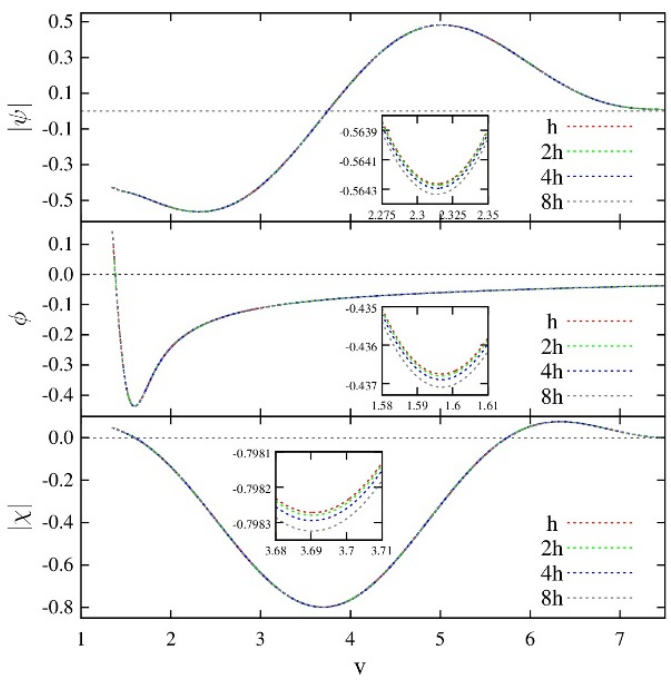

(a)

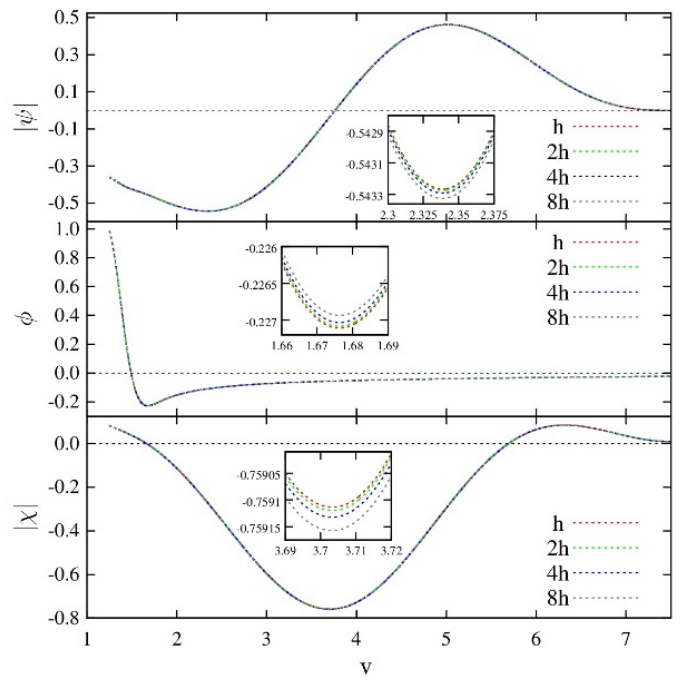

(b)

Figure 26. (color online) The convergence of field functions. The phantom field, $\phi$, and the moduli of complex scalar fields, $|\psi|$ and $|\chi|$, were plotted versus $v$ for evolutions conducted with integration steps, which were multiples of $h=10^{-4}$, along hypersurfaces of constant $u$ equal to (a) 1.3504 for evolution 1 and (b) 1.2504 for evolution 2 .

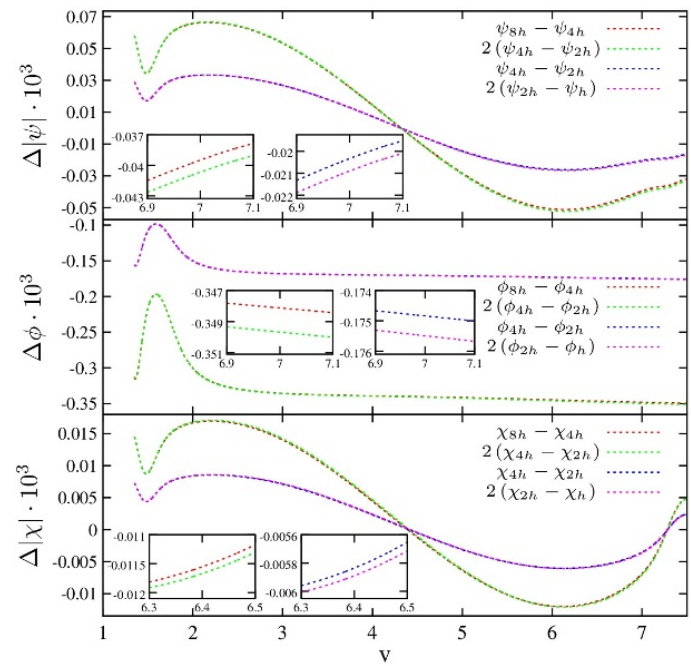

(a)

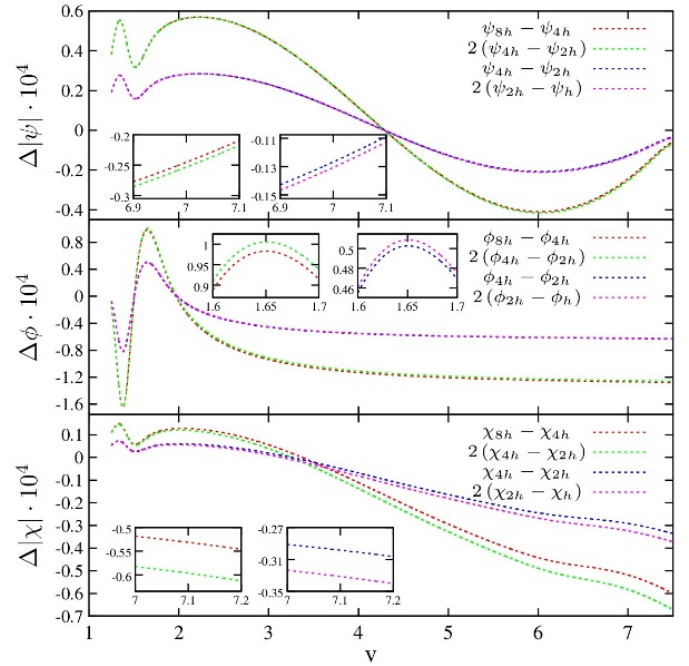

(b)

Figure 27. (color online) The linear convergence of the code. The differences between the phantom field functions, $\Delta \phi$, and the moduli of complex scalar fields, $\Delta|\psi|$ and $\Delta|\chi|$, calculated on grids with different integration steps (multiples of $h=10^{-4}$ ) and their doubles were obtained along the same hypersurfaces of constant $u$ as in figure 26 for (a) evolution 1 and (b) evolution 2 . 


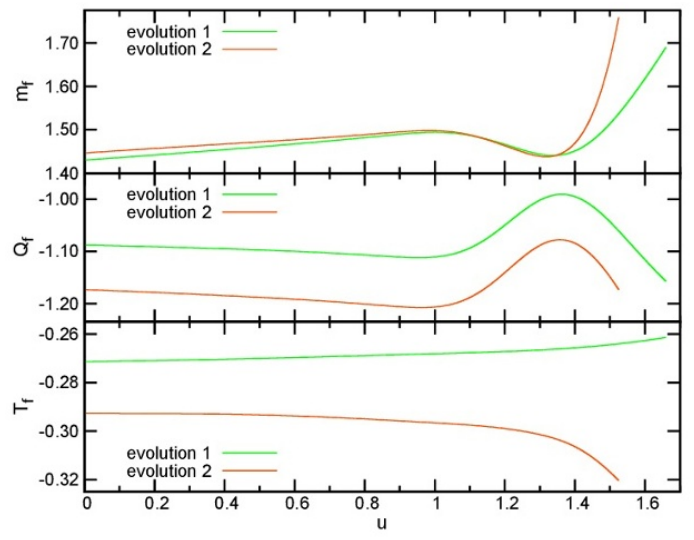

Figure 28. (color online) Hawking masses, $m_{f}$, and charges related to the U(1)-gauge fields, $Q_{f}$ and $T_{f}$, calculated along $v_{f}=7.5$ as functions of retarded time, $u$, for both tested evolutions.

The second test of the numerical code was based on checking the mass and charge conservation in the spacetime. The Hawking mass (3.4) and the charges related to both complex scalar fields coupled to the U(1)-gauge fields (2.14) and (2.17) as functions of retarded time along the line $v=7.5$, which was a maximal value of advanced time achieved numerically, are presented in figure 28 for the investigated evolutions. Since during the process the matter was scattered by the gravitational potential barrier when the collapsing shell approached its gravitational radius, the physical quantities were not conserved during the whole evolution. The effect of the outgoing flux was negligible except for the vicinity of the event horizon. The deviation from the constancy increased with advanced time, as the horizon was approached. The maximal percentage deviations from the particular quantity conservation up to $u=1.25$ were equal to $3.3 \%, 8.1 \%$ and $1.1 \%$ for the mass, the electric charge and the charge associated with the dark matter sector, respectively. The analysis of mass and charge conservation in spacetime led to the conclusion that the behavior of matter investigated numerically was correct within the domain of integration.

Open Access. This article is distributed under the terms of the Creative Commons Attribution License (CC-BY 4.0), which permits any use, distribution and reproduction in any medium, provided the original author(s) and source are credited.

\section{References}

[1] R.C. Tolman, Static solutions of Einstein's field equations for spheres of fluid, Phys. Rev. 55 (1939) 364 [INSPIRE].

[2] J.R. Oppenheimer and G.M. Volkoff, On massive neutron cores, Phys. Rev. 55 (1939) 374 [INSPIRE].

[3] E. Sorkin and T. Piran, The effects of pair creation on charged gravitational collapse, Phys. Rev. D 63 (2001) 084006 [gr-qc/0009095] [INSPIRE].

[4] E. Sorkin and T. Piran, Formation and evaporation of charged black holes, Phys. Rev. D 63 (2001) 124024 [gr-qc/0103090] [INSPIRE]. 
[5] S. Hod and T. Piran, Mass inflation in dynamical gravitational collapse of a charged scalar field, Phys. Rev. Lett. 81 (1998) 1554 [gr-qc/9803004] [INSPIRE].

[6] Y. Oren and T. Piran, On the collapse of charged scalar fields, Phys. Rev. D 68 (2003) 044013 [gr-qc/0306078] [INSPIRE].

[7] E. Poisson and W. Israel, Internal structure of black holes, Phys. Rev. D 41 (1990) 1796 [INSPIRE].

[8] S.E. Hong, D.-I. Hwang, E.D. Stewart and D.-H. Yeom, The causal structure of dynamical charged black holes, Class. Quant. Grav. 27 (2010) 045014 [arXiv:0808.1709] [INSPIRE].

[9] D.-I. Hwang and D.-H. Yeom, Internal structure of charged black holes, Phys. Rev. D 84 (2011) 064020 [arXiv: 1010.2585] [INSPIRE].

[10] D.-I. Hwang and D.-H. Yeom, Responses of the Brans-Dicke field due to gravitational collapses, Class. Quant. Grav. 27 (2010) 205002 [arXiv: 1002.4246] [INSPIRE].

[11] D.-I. Hwang, B.-H. Lee and D.-H. Yeom, Mass inflation in $f(R)$ gravity: a conjecture on the resolution of the mass inflation singularity, JCAP 12 (2011) 006 [arXiv:1110.0928] [INSPIRE].

[12] J. Hansen and D.-H. Yeom, Charged black holes in string-inspired gravity: I. Causal structures and responses of the Brans-Dicke field, JHEP 10 (2014) 040 [arXiv:1406.0976] [INSPIRE].

[13] J. Hansen and D.-H. Yeom, Charged black holes in string-inspired gravity: II. Mass inflation and dependence on parameters and potentials, JCAP 09 (2015) 019 [arXiv:1506.05689] [INSPIRE].

[14] A. Borkowska, M. Rogatko and R. Moderski, Collapse of charged scalar field in dilaton gravity, Phys. Rev. D 83 (2011) 084007 [arXiv: 1103.4808] [INSPIRE].

[15] A. Nakonieczna and M. Rogatko, Dilatons and the dynamical collapse of charged scalar field, Gen. Rel. Grav. 44 (2012) 3175 [arXiv:1209.3614] [INSPIRE].

[16] A. Nakonieczna, M. Rogatko and R. Moderski, Dynamical collapse of charged scalar field in phantom gravity, Phys. Rev. D 86 (2012) 044043 [arXiv:1209.1203] [INSPIRE].

[17] Planck collaboration, P.A.R. Ade et al., Planck 2013 results. XVI. Cosmological parameters, Astron. Astrophys. 571 (2014) A16 [arXiv:1303.5076] [INSPIRE].

[18] R.-G. Cai and A. Wang, Black hole formation from collapsing dust fluid in a background of dark energy, Phys. Rev. D 73 (2006) 063005 [astro-ph/0505136] [INSPIRE].

[19] S. Chakraborty and T. Bandyopadhyay, Collapse dynamics of a star of dark matter and dark energy, Grav. Cosmol. 16 (2010) 151 [gr-qc/0609038] [INSPIRE].

[20] P. Rudra and U. Debnath, Gravitational collapse with dark energy and dark matter in Hořava-Lifshitz gravity, Int. J. Theor. Phys. 53 (2014) 2668 [arXiv:1307.5823] [InSPIRE].

[21] Q. Wang and Z. Fan, Dynamical evolution of quintessence dark energy in collapsing dark matter halos, Phys. Rev. D 79 (2009) 123012 [arXiv: 0906.3349] [INSPIRE].

[22] D.F. Mota and C. van de Bruck, On the spherical collapse model in dark energy cosmologies, Astron. Astrophys. 421 (2004) 71 [astro-ph/0401504] [INSPIRE].

[23] M. Le Delliou and T. Barreiro, Interacting dark energy collapse with matter components separation, JCAP 02 (2013) 037 [arXiv:1208.6373] [INSPIRE]. 
[24] T.R.P. Caramês, J.C. Fabris and H.E.S. Velten, Spherical collapse for unified dark matter models, Phys. Rev. D 89 (2014) 083533 [arXiv:1401.5608] [INSPIRE].

[25] J. Fuller and C. Ott, Dark matter-induced collapse of neutron stars: a possible link between fast radio bursts and the missing pulsar problem, Mon. Not. Roy. Astron. Soc. 450 (2015) L71 [arXiv:1412.6119] [INSPIRE].

[26] D.R. Lorimer, M. Bailes, M.A. McLaughlin, D.J. Narkevic and F. Crawford, A bright millisecond radio burst of extragalactic origin, Science 318 (2007) 777 [arXiv:0709.4301] [INSPIRE].

[27] S. Johnston et al., Discovery of two pulsars towards the galactic centre, Mon. Not. Roy. Astron. Soc. 373 (2006) L6 [astro-ph/0606465] [INSPIRE].

[28] J.-H. Choi, I. Shlosman and M.C. Begelman, Supermassive black hole formation at high redshifts via direct collapse in a cosmological context, Mon. Not. Roy. Astron. Soc. 450 (2015) 4411 [arXiv: 1412.2761] [INSPIRE].

[29] I. Shlosman, J.-H. Choi, M.C. Begelman and K. Nagamine, Supermassive black hole seed formation at high redshifts: long-term evolution of the direct collapse, arXiv:1508.05098 [INSPIRE].

[30] F. Zwicky, Die Rotverschiebung von extragalaktischen Nebeln (in German), Helv. Phys. Acta 6 (1933) 110 [INSPIRE].

[31] V.C. Rubin and W.K. Ford, Jr., Rotation of the Andromeda nebula from a spectroscopic survey of emission regions, Astrophys. J. 159 (1970) 379 [INSPIRE].

[32] Planck collaboration, P.A.R. Ade et al., Planck 2013 results. XVII. Gravitational lensing by large-scale structure, Astron. Astrophys. 571 (2014) A17 [arXiv:1303.5077] [INSPIRE].

[33] S.W. Randall, M. Markevitch, D. Clowe, A.H. Gonzalez and M. Bradac, Constraints on the self-interaction cross-section of dark matter from numerical simulations of the merging galaxy cluster $1 E 0657$ - 56, Astrophys. J. 679 (2008) 1173 [arXiv:0704.0261] [INSPIRE].

[34] D. Harvey, R. Massey, T. Kitching, A. Taylor and E. Tittley, The non-gravitational interactions of dark matter in colliding galaxy clusters, Science $\mathbf{3 4 7}$ (2015) 1462 [arXiv: 1503.07675] [INSPIRE].

[35] R. Massey et al., The behaviour of dark matter associated with four bright cluster galaxies in the $10 \mathrm{kpc}$ core of Abell 3827, Mon. Not. Roy. Astron. Soc. 449 (2015) 3393 [arXiv: 1504.03388] [INSPIRE].

[36] D. Hooper and L. Goodenough, Dark matter annihilation in the galactic center as seen by the Fermi gamma ray space telescope, Phys. Lett. B 697 (2014) 412 [arXiv:1010.2752] [INSPIRE].

[37] K.N. Abazajian and M. Kaplinghat, Detection of a gamma-ray source in the galactic center consistent with extended emission from dark matter annihilation and concentrated astrophysical emission, Phys. Rev. D 86 (2012) 083511 [Erratum ibid. D 87 (2013) 129902] [arXiv:1207.6047] [INSPIRE].

[38] C. Gordon and O. Macias, Dark matter and pulsar model constraints from galactic center Fermi-LAT gamma ray observations, Phys. Rev. D 88 (2013) 083521 [Erratum ibid. D 89 (2014) 049901] [arXiv:1306.5725] [INSPIRE]. 
[39] E. Bulbul, M. Markevitch, A. Foster, R.K. Smith, M. Loewenstein and S.W. Randall, Detection of an unidentified emission line in the stacked $X$-ray spectrum of galaxy clusters, Astrophys. J. 789 (2014) 13 [arXiv:1402.2301] [INSPIRE].

[40] A. Boyarsky, O. Ruchayskiy, D. Iakubovskyi and J. Franse, Unidentified line in X-ray spectra of the Andromeda galaxy and Perseus galaxy cluster, Phys. Rev. Lett. 113 (2014) 251301 [arXiv: 1402.4119] [INSPIRE].

[41] V.A. Mitsou, Shedding light on dark matter at colliders, Int. J. Mod. Phys. A 28 (2013) 1330052 [arXiv: 1310.1072] [INSPIRE].

[42] D. Abercrombie et al., Dark matter benchmark models for early LHC run-2 searches: report of the ATLAS/CMS dark matter forum, arXiv:1507.00966 [INSPIRE].

[43] J. Bramante and T. Linden, Detecting dark matter with imploding pulsars in the galactic center, Phys. Rev. Lett. 113 (2014) 191301 [arXiv:1405.1031] [INSPIRE].

[44] I. Lopes and J. Silk, A particle dark matter footprint on the first generation of stars, Astrophys. J. 786 (2014) 25 [arXiv: 1404.3909] [INSPIRE].

[45] C.-F. Chang, E. Ma and T.-C. Yuan, Multilepton Higgs decays through the dark portal, JHEP 03 (2014) 054 [arXiv:1308.6071] [INSPIRE].

[46] Heavy Photon Search Experiment webpage, https://confluence.slac.stanford.edu/display/hpsg/Heavy+Photon+Search+Experiment.

[47] BABAR collaboration, J.P. Lees et al., Search for a dark photon in $e^{+} e^{-}$collisions at BaBar, Phys. Rev. Lett. 113 (2014) 201801 [arXiv:1406.2980] [INSPIRE].

[48] S. Baek, P. Ko and W.-I. Park, Singlet portal extensions of the standard seesaw models to a dark sector with local dark symmetry, JHEP 07 (2013) 013 [arXiv : 1303.4280] [INSPIRE].

[49] S. Baek, P. Ko and W.-I. Park, An alternative to the standard model, AIP Conf. Proc. 1604 (2014) 73 [arXiv:1310.6229] [INSPIRE].

[50] S. Baek, P. Ko and W.-I. Park, Local $Z_{2}$ scalar dark matter model confronting galactic GeV-scale $\gamma$-ray, Phys. Lett. B 747 (2015) 255 [arXiv: 1407.6588] [inSPIRE].

[51] Ł. Nakonieczny and M. Rogatko, Analytic study on backreacting holographic superconductors with dark matter sector, Phys. Rev. D 90 (2014) 106004 [arXiv:1411.0798] [INSPIRE].

[52] Ł. Nakonieczny, M. Rogatko and K.I. Wysokiński, Magnetic field in holographic superconductor with dark matter sector, Phys. Rev. D 91 (2015) 046007 [arXiv: 1502.02550] [INSPIRE].

[53] Ł. Nakonieczny, M. Rogatko and K.I. Wysokiński, Analytic investigation of holographic phase transitions influenced by dark matter sector, Phys. Rev. D 92 (2015) 066008 [arXiv: 1509.01769] [INSPIRE].

[54] Supernova Cosmology Project collaboration, S. Perlmutter et al., Measurements of $\Omega$ and $\Lambda$ from 42 high redshift supernovae, Astrophys. J. 517 (1999) 565 [astro-ph/9812133] [INSPIRE].

[55] Supernova Search Team collaboration, A.G. Riess et al., Observational evidence from supernovae for an accelerating universe and a cosmological constant, Astron. J. 116 (1998) 1009 [astro-ph/9805201] [INSPIRE]. 
[56] Supernova Search Team collaboration, B.P. Schmidt et al., The high $Z$ supernova search: measuring cosmic deceleration and global curvature of the universe using type-IA supernovae, Astrophys. J. $\mathbf{5 0 7}$ (1998) 46 [astro-ph/9805200] [INSPIRE].

[57] Supernova Search Team collaboration, J.L. Tonry et al., Cosmological results from high-Z supernovae, Astrophys. J. 594 (2003) 1 [astro-ph/0305008] [INSPIRE].

[58] R.R. Caldwell and M. Kamionkowski, The physics of cosmic acceleration, Ann. Rev. Nucl. Part. Sci. 59 (2009) 397 [arXiv: 0903. 0866] [inSPIRE].

[59] R.R. Caldwell, A phantom menace?, Phys. Lett. B 545 (2002) 23 [astro-ph/9908168] [INSPIRE].

[60] G.W. Gibbons and D.A. Rasheed, Dyson pairs and zero mass black holes, Nucl. Phys. B 476 (1996) 515 [hep-th/9604177] [INSPIRE].

[61] G. Clément, J.C. Fabris and M.E. Rodrigues, Phantom black holes in Einstein-Maxwell-dilaton theory, Phys. Rev. D 79 (2009) 064021 [arXiv:0901.4543] [INSPIRE].

[62] M. Azreg-Aïnou, G. Clément, J.C. Fabris and M.E. Rodrigues, Phantom black holes and -models, Phys. Rev. D 83 (2011) 124001 [arXiv:1102.4093] [InSPIRE].

[63] J.A. Wheeler, Geometrodynamics, Academic Press, New York U.S.A. (1963).

[64] M.S. Morris and K.S. Thorne, Wormholes in space-time and their use for interstellar travel: a tool for teaching general relativity, Am. J. Phys. 56 (1988) 395 [InSPIRE].

[65] F.S.N. Lobo, F. Parsaei and N. Riazi, New asymptotically flat phantom wormhole solutions, Phys. Rev. D 87 (2013) 084030 [arXiv:1212.5806] [INSPIRE].

[66] J.P.S. Lemos, F.S.N. Lobo and S. Quinet de Oliveira, Morris-Thorne wormholes with a cosmological constant, Phys. Rev. D 68 (2003) 064004 [gr-qc/0302049] [INSPIRE].

[67] F.S.N. Lobo and M.A. Oliveira, General class of vacuum Brans-Dicke wormholes, Phys. Rev. D 81 (2010) 067501 [arXiv: 1001.0995] [INSPIRE].

[68] E.F. Eiroa and C. Simeone, Thin-shell wormholes in dilaton gravity, Phys. Rev. D 71 (2005) 127501 [gr-qc/0502073] [inSPIRE].

[69] J.A. González, F.S. Guzmán, N. Montelongo-García and T. Zannias, On wormholes supported by phantom energy, Phys. Rev. D 79 (2009) 064027 [arXiv:0906.5590] [INSPIRE].

[70] S.V. Sushkov, Wormholes supported by a phantom energy, Phys. Rev. D 71 (2005) 043520 [gr-qc/0502084] [INSPIRE].

[71] A. Das and S. Kar, The Ellis wormhole with 'tachyon matter', Class. Quant. Grav. 22 (2005) 3045 [gr-qc/0505124] [INSPIRE].

[72] A.B. Balakin, J.P.S. Lemos and A.E. Zayats, Nonminimal coupling for the gravitational and electromagnetic fields: traversable electric wormholes, Phys. Rev. D 81 (2010) 084015 [arXiv: 1003.4584] [INSPIRE].

[73] P. Kanti, B. Kleihaus and J. Kunz, Wormholes in dilatonic Einstein-Gauss-Bonnet theory, Phys. Rev. Lett. 107 (2011) 271101 [arXiv:1108.3003] [InSPIRE].

[74] P. Kanti, B. Kleihaus and J. Kunz, Stable Lorentzian wormholes in dilatonic Einstein-Gauss-Bonnet theory, Phys. Rev. D 85 (2012) 044007 [arXiv:1111.4049] [INSPIRE]. 
[75] T. Harko, F.S.N. Lobo, M.K. Mak and S.V. Sushkov, Modified-gravity wormholes without exotic matter, Phys. Rev. D 87 (2013) 067504 [arXiv:1301.6878] [InSPIRE].

[76] M.R. Mehdizadeh, M.K. Zangeneh and F.S.N. Lobo, Einstein-Gauss-Bonnet traversable wormholes satisfying the weak energy condition, Phys. Rev. D 91 (2015) 084004 [arXiv: 1501.04773] [INSPIRE].

[77] M. Cataldo and P. Meza, Phantom evolving wormholes with big rip singularities, Phys. Rev. D 87 (2013) 064012 [arXiv: 1302.3748] [INSPIRE].

[78] M.K. Zangeneh, F.S.N. Lobo and N. Riazi, Higher-dimensional evolving wormholes satisfying the null energy condition, Phys. Rev. D 90 (2014) 024072 [arXiv:1406.5703] [INSPIRE].

[79] ALICE collaboration, Production of $\Sigma(1385)^{ \pm}$and $\Xi(1530)^{0}$ in proton-proton collisions at $\sqrt{s}=7$ TeV, Eur. Phys. J. C 75 (2015) 1 [arXiv:1406.3206] [InSPIRE].

[80] T. Damour and S.N. Solodukhin, Wormholes as black hole foils, Phys. Rev. D 76 (2007) 024016 [arXiv: 0704.2667] [INSPIRE].

[81] C. Bambi, Can the supermassive objects at the centers of galaxies be traversable wormholes? The first test of strong gravity for $\mathrm{mm} /$ sub-mm very long baseline interferometry facilities, Phys. Rev. D 87 (2013) 107501 [arXiv:1304.5691] [INSPIRE].

[82] Z. Li and C. Bambi, Distinguishing black holes and wormholes with orbiting hot spots, Phys. Rev. D 90 (2014) 024071 [arXiv: 1405.1883] [InSPIRE].

[83] K.S. Virbhadra and G.F.R. Ellis, Schwarzschild black hole lensing, Phys. Rev. D 62 (2000) 084003 [astro-ph/9904193] [INSPIRE].

[84] K.S. Virbhadra and G.F.R. Ellis, Gravitational lensing by naked singularities, Phys. Rev. D 65 (2002) 103004 [INSPIRE].

[85] K.S. Virbhadra and C.R. Keeton, Time delay and magnification centroid due to gravitational lensing by black holes and naked singularities, Phys. Rev. D 77 (2008) 124014 [arXiv:0710.2333] [INSPIRE].

[86] K.S. Virbhadra, Relativistic images of Schwarzschild black hole lensing, Phys. Rev. D 79 (2009) 083004 [arXiv:0810.2109] [INSPIRE].

[87] Z. Kovács and T. Harko, Can accretion disk properties observationally distinguish black holes from naked singularities?, Phys. Rev. D 82 (2010) 124047 [arXiv:1011.4127] [INSPIRE].

[88] P.S. Joshi, D. Malafarina and R. Narayan, Distinguishing black holes from naked singularities through their accretion disc properties, Class. Quant. Grav. 31 (2014) 015002 [arXiv: 1304.7331] [INSPIRE].

[89] S. Sahu, M. Patil, D. Narasimha and P.S. Joshi, Can strong gravitational lensing distinguish naked singularities from black holes?, Phys. Rev. D 86 (2012) 063010 [arXiv:1206.3077] [INSPIRE].

[90] M. Rakhmanov, Dilaton black holes with electric charge, Phys. Rev. D 50 (1994) 5155 [hep-th/9310174] [INSPIRE].

[91] A. Gupta, R. Primulando and P. Saraswat, A new probe of dark sector dynamics at the LHC, JHEP 09 (2015) 079 [arXiv: 1504.01385] [INSPIRE]. 
[92] J. Suzuki, T. Horie, Y. Inoue and M. Minowa, Experimental search for hidden photon CDM in the eV mass range with a dish antenna, JCAP 09 (2015) 042 [arXiv:1504.00118] [INSPIRE].

[93] H.K. Dreiner, J.-F. Fortin, C. Hanhart and L. Ubaldi, Supernova constraints on MeV dark sectors from $e^{+} e^{-}$annihilations, Phys. Rev. D 89 (2014) 105015 [arXiv:1310.3826] [INSPIRE].

[94] J. Blümlein and J. Brunner, New exclusion limits for dark gauge forces from beam-dump data, Phys. Lett. B 701 (2011) 155 [arXiv:1104.2747] [InSPIRE].

[95] J. Blümlein and J. Brunner, New exclusion limits on dark gauge forces from proton bremsstrahlung in beam-dump data, Phys. Lett. B 731 (2014) 320 [arXiv:1311.3870] [INSPIRE].

[96] ATLAS collaboration, Search for long-lived neutral particles decaying into lepton jets in proton-proton collisions at $\sqrt{s}=8 \mathrm{TeV}$ with the ATLAS detector, JHEP 11 (2014) 088 [arXiv: 1409.0746] [INSPIRE].

[97] S.N. Gninenko, Constraints on sub-GeV hidden sector gauge bosons from a search for heavy neutrino decays, Phys. Lett. B 713 (2012) 244 [arXiv:1204.3583] [INSPIRE].

[98] A. Mirizzi, J. Redondo and G. Sigl, Microwave background constraints on mixing of photons with hidden photons, JCAP 03 (2009) 026 [arXiv:0901.0014] [INSPIRE].

[99] A. Afanasev et al., New experimental limit on photon hidden-sector paraphoton mixing, Phys. Lett. B 679 (2009) 317 [arXiv:0810.4189] [InSPIRE].

[100] KLOE-2 collaboration, F. Archilli et al., Search for a vector gauge boson in $\phi$ meson decays with the KLOE detector, Phys. Lett. B 706 (2014) 251 [arXiv:1110.0411] [INSPIRE].

[101] KLOE-2 collaboration, D. Babusci et al., Limit on the production of a light vector gauge boson in $\phi$ meson decays with the KLOE detector, Phys. Lett. B 720 (2013) 111 [arXiv: 1210.3927] [INSPIRE].

[102] WASA-AT-COSY collaboration, P. Adlarson et al., Search for a dark photon in the $\pi^{0} \rightarrow e^{+} e^{-} \gamma$ decay, Phys. Lett. B 726 (2013) 187 [arXiv:1304.0671] [INSPIRE].

[103] APEX collaboration, S. Abrahamyan et al., Search for a new gauge boson in electron-nucleus fixed-target scattering by the APEX experiment, Phys. Rev. Lett. 107 (2011) 191804 [arXiv:1108.2750] [INSPIRE].

[104] A1 collaboration, H. Merkel et al., Search for light gauge bosons of the dark sector at the Mainz microtron, Phys. Rev. Lett. 106 (2011) 251802 [arXiv:1101.4091] [INSPIRE].

[105] HADES collaboration, G. Agakishiev et al., Searching a dark photon with HADES, Phys. Lett. B 731 (2014) 265 [arXiv: 1311.0216] [INSPIRE].

[106] T. Ortín, Gravity and strings, Cambridge University Press, Cambridge U.K. (2004).

[107] E. Alvarez and J. Conde, Are the string and Einstein frames equivalent, Mod. Phys. Lett. A 17 (2002) 413 [gr-qc/0111031] [INSPIRE].

[108] G. Veneziano, String cosmology: the pre-big bang scenario, in The primordial universe L'univers primordial, P. Binétruy, R. Schaeffer, J. Silk and F. David eds., Les Houches Ecole d'Ete de Physique Theorique 71, Springer, Berlin Heidelberg Germany (2002), pg. 581. 
[109] É.É. Flanagan, Palatini form of 1/R gravity, Phys. Rev. Lett. 92 (2004) 071101 [astro-ph/0308111] [INSPIRE].

[110] D.N. Vollick, On the viability of the Palatini form of $1 / R$ gravity, Class. Quant. Grav. 21 (2004) 3813 [gr-qc/0312041] [INSPIRE].

[111] G. Domènech and M. Sasaki, Conformal frame dependence of inflation, JCAP 04 (2015) 022 [arXiv: 1501.07699] [INSPIRE].

[112] J. White, M. Minamitsuji and M. Sasaki, Non-linear curvature perturbation in multi-field inflation models with non-minimal coupling, JCAP 09 (2013) 015 [arXiv:1306.6186] [INSPIRE].

[113] T. Chiba and M. Yamaguchi, Conformal-frame (in)dependence of cosmological observations in scalar-tensor theory, JCAP 10 (2013) 040 [arXiv: 1308.1142] [INSPIRE].

[114] C.W. Misner, K.S. Thorne and J.A. Wheeler, Gravitation, W.H. Freeman and Company, U.S.A. (1973).

[115] R.A. d'Inverno and J. Smallwood, Covariant $2+2$ formulation of the initial-value problem in general relativity, Phys. Rev. D 22 (1980) 1233 [InSPIRE].

[116] R.S. Hamadé and J.M. Stewart, The spherically symmetric collapse of a massless scalar field, Class. Quant. Grav. 13 (1996) 497 [gr-qc/9506044] [INSPIRE].

[117] S. Ayal and T. Piran, Spherical collapse of a massless scalar field with semiclassical corrections, Phys. Rev. D 56 (1997) 4768 [gr-qc/9704027] [InSPIRE].

[118] M.W. Choptuik, Universality and scaling in gravitational collapse of a massless scalar field, Phys. Rev. Lett. 70 (1993) 9 [INSPIRE].

[119] V.P. Frolov and I.D. Novikov, Black hole physics: basic concepts and new developments, Kluwer Academic Publishers, The Netherlands (1998).

[120] S. Hawking, Gravitational radiation in an expanding universe, J. Math. Phys. 9 (1968) 598 [INSPIRE].

[121] D. Hochberg and M. Visser, Dynamic wormholes, anti-trapped surfaces and energy conditions, Phys. Rev. D 58 (1998) 044021 [gr-qc/9802046] [InSPIRE].

[122] D. Hochberg and M. Visser, The null energy condition in dynamic wormholes, Phys. Rev. Lett. 81 (1998) 746 [gr-qc/9802048] [INSPIRE].

[123] H. Maeda, T. Harada and B.J. Carr, Cosmological wormholes, Phys. Rev. D 79 (2009) 044034 [arXiv: 0901.1153] [INSPIRE].

[124] S.A. Hayward, Dynamic wormholes, Int. J. Mod. Phys. D 8 (1999) 373 [gr-qc/9805019] [INSPIRE].

[125] S.A. Hayward, Wormhole dynamics in spherical symmetry, Phys. Rev. D 79 (2009) 124001 [arXiv: 0903.5438] [INSPIRE].

[126] H.-A. Shinkai and S.A. Hayward, Fate of the first traversible wormhole: black hole collapse or inflationary expansion, Phys. Rev. D 66 (2002) 044005 [gr-qc/0205041] [INSPIRE].

[127] H. Koyama, S.A. Hayward and S.-W. Kim, Construction and enlargement of dilatonic wormholes by impulsive radiation, Phys. Rev. D 67 (2003) 084008 [gr-qc/0212106] [INSPIRE]. 
[128] H. Koyama and S.A. Hayward, Construction and enlargement of traversable wormholes from Schwarzschild black holes, Phys. Rev. D 70 (2004) 084001 [gr-qc/0406113] [INSPIRE].

[129] H. Okawa, V. Cardoso and P. Pani, Collapse of self-interacting fields in asymptotically flat spacetimes: do self-interactions render Minkowski spacetime unstable?, Phys. Rev. D 89 (2014) 041502 [arXiv:1311.1235] [INSPIRE].

[130] T. Harada, H. Maeda and B.J. Carr, Non-existence of self-similar solutions containing a black hole in a universe with a stiff fluid or scalar field or quintessence, Phys. Rev. D 74 (2006) 024024 [astro-ph/0604225] [INSPIRE].

[131] B.J. Carr, K. Kohri, Y. Sendouda and J. Yokoyama, New cosmological constraints on primordial black holes, Phys. Rev. D 81 (2010) 104019 [arXiv:0912.5297] [INSPIRE].

[132] A. Einstein and E.G. Straus, The influence of the expansion of space on the gravitation fields surrounding the individual stars, Rev. Mod. Phys. 17 (1945) 120 [InSPIRE].

[133] G.C. McVittie, The mass-particle in an expanding universe, Mon. Not. Roy. Astron. Soc. 93 (1933) 325 [INSPIRE].

[134] D. Kastor and J.H. Traschen, Cosmological multi-black hole solutions, Phys. Rev. D 47 (1993) 5370 [hep-th/9212035] [INSPIRE].

[135] G.W. Gibbons and K.-I. Maeda, Black holes in an expanding universe, Phys. Rev. Lett. 104 (2010) 131101 [arXiv:0912.2809] [INSPIRE].

[136] M. Rogatko, Dilaton black holes on thick branes, Phys. Rev. D 64 (2001) 064014 [hep-th/0110018] [INSPIRE].

[137] M. Rogatko, Cosmological black holes on branes, Phys. Rev. D 69 (2004) 044022 [hep-th/0312105] [INSPIRE].

[138] K.-I. Maeda and M. Nozawa, Black hole in the expanding universe from intersecting branes, Phys. Rev. D 81 (2010) 044017 [arXiv:0912.2811] [INSPIRE].

[139] S. Chadburn and R. Gregory, Time dependent black holes and scalar hair, Class. Quant. Grav. 31 (2014) 195006 [arXiv:1304.6287] [INSPIRE]. 